\title{
Time domain model and experimental validation of non-contact surface wave scanner
}

\author{
$\mathrm{Ji} \mathrm{LI}^{\mathrm{a}, \mathrm{b}}$, Bogdan PIWAKOWSKI ${ }^{\mathrm{b}}$ \\ ${ }^{a}$ Nuclear Power Institute of China, 610213, Chengdu, China \\ ${ }^{\mathrm{b}}$ IEMN TPIA UMR CNRS 8520, Ecole Centrale de Lille, BP 4859651 Villeneuve d'Ascq \\ Cedex, France. \\ liji2615@outlook.com; bogdan.piwakowski@ec-lille.fr
}

\begin{abstract}
\end{abstract}
This paper presents a time-domain model for the prediction of acoustic field in an air-coupled, non-contact, ultrasonic surface wave scanner, which includes an air-coupled Emitter, the Propagation space, and an air-coupled Receiver (EPR). The computation is divided into three steps, with each step being modeled in the time domain by its spatio-temporal transfer function. The latter are then used in turn, to find the pulse response of the overall system.

The model takes the finite size of the aperture receiver, the attenuation in both air and the tested solid sample, as well as the electric response of the emitter-receiver set $h_{e}$ into account. The attenuation is characterized by a causal time-domain Green's function, allowing wideband attenuation of a lossy medium, obeying the power law $\alpha(\omega)=\alpha_{0} \omega^{\eta}, 0 \leq \eta \leq 2$, to be used. The model is implemented numerically using a Discrete Representation approach. It is then validated quantitatively by comparing the predicted acoustic field with experiment. The prediction error for three typical field features, the system's impulse response, the on-axis field distribution, and the directivity pattern, is globally smaller than $3 \%$.

In order to obtain this high level of accuracy in the model, the parameters characterizing the solid sample used during the experiment were measured experimentally, with a specifically developed experimental setup.

Overall, the proposed model is approximately 100 times faster than 3D FEM with an equivalent spatio-temporal resolution. In parallel, a simplified model is proposed, which neglects the attenuation in air and assumes the emitter inclination angle to be perfectly adjusted. This approach makes it possible to further shorten the computational by a factor of 
about ten, whilst maintaining good accuracy. Thanks to its computational efficiency, the proposed model can be used to formulate various recommendations concerning the scanner settings, in particular the inclination angles of the emitter and receiver, and their distance from the sample.

Keywords air-coupled transducers, lossy media, non-contact ultrasonics, Green's function, impulse response, discrete representation approach

\section{Introduction}

The most commonly used and recognized ultrasonic tests rely on direct contact with the sample, and ultrasound signals are generated and received by means of wet coupling (oil, water, etc.) or mechanical contact.

However, various drawbacks have been observed with direct contact techniques, in particular those arising from temporal variations in coupling quality (especially for porous and liquid absorbing materials) and limitations in the case of fast scanning. These drawbacks have encouraged the development of non-contact techniques, which do not contaminate the surface of the sample under investigation, and enable rapid, automated scanning processes. As a consequence of their straightforward and efficient implementation, non-contact techniques have been applied to automatic, fast, non-destructive testing in different engineering disciplines, e.g. for the testing of paper, composites, food, wooden materials, metal plates, as well as the evaluation of surface roughness, and the inspection of concrete. A complete review of various air-coupled, non-destructive testing approaches and applications is provided in two recent papers $[1,2]$. Further examples are also provided in [3-9]. The above techniques are not restricted to classical geometries, i.e. pulse echo, or the transmission of longitudinal waves[10, 11], but can also make use of surface or Lamb waves, in particular for non-destructive testing (NDT).

Surface waves (SW) have been widely used for NDT, in different fields. This type of wave propagates along the surface of the tested sample, and penetrates down to a depth close to the signal wavelength [12], thus providing valuable information related to the near surface properties, such as micro-cracking, surface defects, dis-bonding, surface layering, etc. As they travel along the surface of the material, experiencing cylindrical divergence, SWs propagate over a greater distance than bulk waves, and thus enable the inspection of larger structures. 
Concerning non-contact SW setups, one can distinguish between "semi" contact methods, in which either the receiver or emitter is of the contact type, and "fully" non-contact methods in which both are of the non-contact type. Examples of the first group are given by Abraham et al. [13] and Michaels et al. [14], who used a contact SW emitter and laser interferometry for the receiver. Zhu and Popovics [15], and Bjurstrom et al. [16] used a mechanical impactor as the emitter, and by introducing a set of microphones spaced along one profile, the recorded signals enabled multi-channel analysis of surface or Lamb waves. Kim et al. [17] applied the semi-contact technique to pipeline testing, using a contact emitter and a non-contact receiver. A "fully" non-contact technique which does not contaminate the surface of the sample under investigation, and enables rapid and automated scanning through the use of Lamb waves, has recently been used for NDT of composites. The authors used a non-contact piezo-ceramic emitter and a laser interferometer for the receiver $[18,19]$.

In the case of SW emission, an angle beam transducer, which is typically an assembly of a plastic wedge and a piezo-electric transducer, can be used for efficient SW emission and reception [20]. This approach has several advantages in that SWs are preferentially excited, their direction of propagation and directivity are controllable, and in pulse mode the SW pulse maintains the same shape [21]. The solid wedge can be replaced by a liquid, thus leading to the so-called liquid wedge technique. This method allows time-consuming coupling operations to be avoided, and produces repeatable SW signal levels [21, 22]. In the non-contact version, the liquid is simply replaced by air. The air-coupled wedge requires no modification/adaptation of the inspected surface, and the wedge angle can easily be adjusted in order to generate a SW or a certain Lamb wave mode. As a consequence of the flexibility of this technique, the air-coupled generation and reception of SW and Lamb waves has received considerable attention in the context of NDT applications.

Examples of the application of a fully non-contact technique, in which both emitter and receiver are air-coupled wedge transducers, are given by the set-ups described in [23-27] for the testing of composites, and by the SW non-contact scanner presented in $[13,28]$. This scanner has been used in practical situations since 2006, and was used to various research projects in civil engineering [29-32], for applications involving concrete cover characterization and crack detection. It has also been used with Lamb waves, for damage detection and localization in composites [33], and to examine railway lines [34].

Experience has shown that the performance of the aforementioned type of SW scanner is strongly influenced by its operating parameters, such as transducer dimensions and inclination, transducer-specimen distance, excitation signal shape and bandwidth, etc. Similar observations 
have also been reported in [35]. In order to clarify the role of these parameters and to establish recommendations for the optimal operation of a SW scanner, an accurate modeling tool is needed for this type of non-contact device.

Several researchers have modeled "full" non-contact Lamb wave testing devices. Castaings and Cawley [23] modeled the Lamb wave non-contact system using the finite element method (FEM). Although Ke et al. [24] also used FEM and implemented a 3D simulation of the system, propagation of the waves in air was neglected. A similar study was made by Fan et al. [36] and Delrue et al. [37], with accurate modeling of wave propagation in air included. In Fan's work, the influence of the non-contact wedge angle on the Lamb signal radiation was also introduced.

The above studies have shown FEM to be an effective tool for the understanding and modeling of an air-coupled system. However, FEM is a highly time-consuming. In addition, these studies pay more attention to the validation of the FEM model itself, rather than to the optimization of the system performance (perhaps as a consequence of the poor computational efficiency of FEM for 3D applications). In addition, they focus mainly on Lamb wave modeling. Among other approaches that have been published, the hybrid method developed in [38] combines the pulse response approach with an analytical solution, making it possible to model a non-contact Lamb wave device. Although the Distributed Point Source Method ([39-42]) was successfully used for accurate modeling of the ultrasonic field produced by plates immerged in a fluid, this technique appears to be more suitable for lossless media, and as far as we are aware has not been applied to air-coupled scenarios.

Finally, despite the above advances, there is at present a lack of suitable, simple and rapid modeling and characterization of the fully non-contact, acoustic SW scanner. The aim of this paper is thus to propose such a modeling tool, which could be used for performance optimization of this type of scanner.

In an air-coupled, non-contact ultrasonic testing system, the ultrasonic waves propagating through a characterized medium are radiated and received by a pair of air-coupled ultrasonic transducers. A crucial requirement for the appropriate understanding and optimization of such a system is the accurate characterization of the basic setup, including the air-coupled Emitter, the Propagation space, and the air-coupled Receiver (EPR). This research was carried out by $\mathrm{Li}$ and Piwakowski [9], and their paper provides several recommendations for accurate modeling of the EPR. This reference shows that very accurate modeling of the EPR can be achieved using Rayleigh's integral, and through correct modeling of the attenuation in air and the finite dimensions of the receiver. The basic components of the solutions developed in [9] 
have thus been used in the present research and for simplicity in the present paper, the latter study is referred to as $R E F$. The computational tool developed in $R E F$ was extended, in order to obtain a complete model of a fully non-contact SW scanner, which includes the non-contact Emitter, the Solid material sample (i.e. tested material) and the non-contact Receiver (ESR).

The computation includes three steps, each of which is modeled in the time domain using Rayleigh's integral and the causal Green's function for a lossy medium. This makes it possible to take the attenuation in air and the tested solid sample into account. The finite receiver size, which has been shown to influence air-coupled measurements, is also modeled.

The originality of this work lies in the sequential use of a set of Rayleigh integrals adapted to a lossy medium, thus allowing each element of the ESR system to be modeled by its own transfer function. These are in turn used to find the pulse response of the overall system.

This paper is organized as follows. Section 2 presents the mathematical model of the complete non-contact scanner. A simplified and more computationally efficient (although slightly less accurate) model was developed in parallel, and is presented in Appendix B. The experimental setup used to validate the models is presented in Sections 3 and 4 . The experiments were performed using a Plexiglas block as the tested solid sample. In order to improve the signal-to-noise ratio (required during the experiments), the chirp technique was used $[10,11,43]$. The solid sample's parameters (attenuation and velocity) were accurately determined using a special procedure described in Appendix A. Finally, the prediction tools developed in this study are used to analyze the influence of typical scanner settings, and in particular to determine the device's sensitivity to the parameters describing the air and the solid (Sections 5, 6, 7).

\section{Time domain model of the ESR system}

The general model of a non-contact SW system is shown Fig.1, where the first block represents the common electric response of the emitter and receiver $h_{e}(t)$ (investigated in $R E F)$ and the second block, characterized by a pressure impulse response $h_{p r}(\mathbf{M}, t)$, represents the modeled system. The system includes the air-coupled Emitter, the Solid sample, and the air-coupled Receiver (ESR). The air-coupled emitter of surface $S_{0}$, excited by an excitation signal $s(t)$, radiates ultrasonic waves into the air. These waves arrive at the surface $S_{1}$ of the solid sample and excite a SW, which propagates along the sample to reach 
the surface $S_{2}$. A certain fraction of the SW energy is re-radiated from $S_{2}$ into the air and is then detected by surface $S_{3}$ of the air-coupled receiver. The proposed ESR model is broken down into the following three steps:

- Step 1 characterizes the radiation/propagation of an acoustic wave from the air-coupled emitter $S_{0}$ to the sample surface $S_{1}$, and is characterized by block $\mathbf{E}$,

- Step 2 describes the SW propagation across the surface of the solid sample from $S_{1}$ to $S_{2}$, and is characterized by block $\mathbf{S}$;

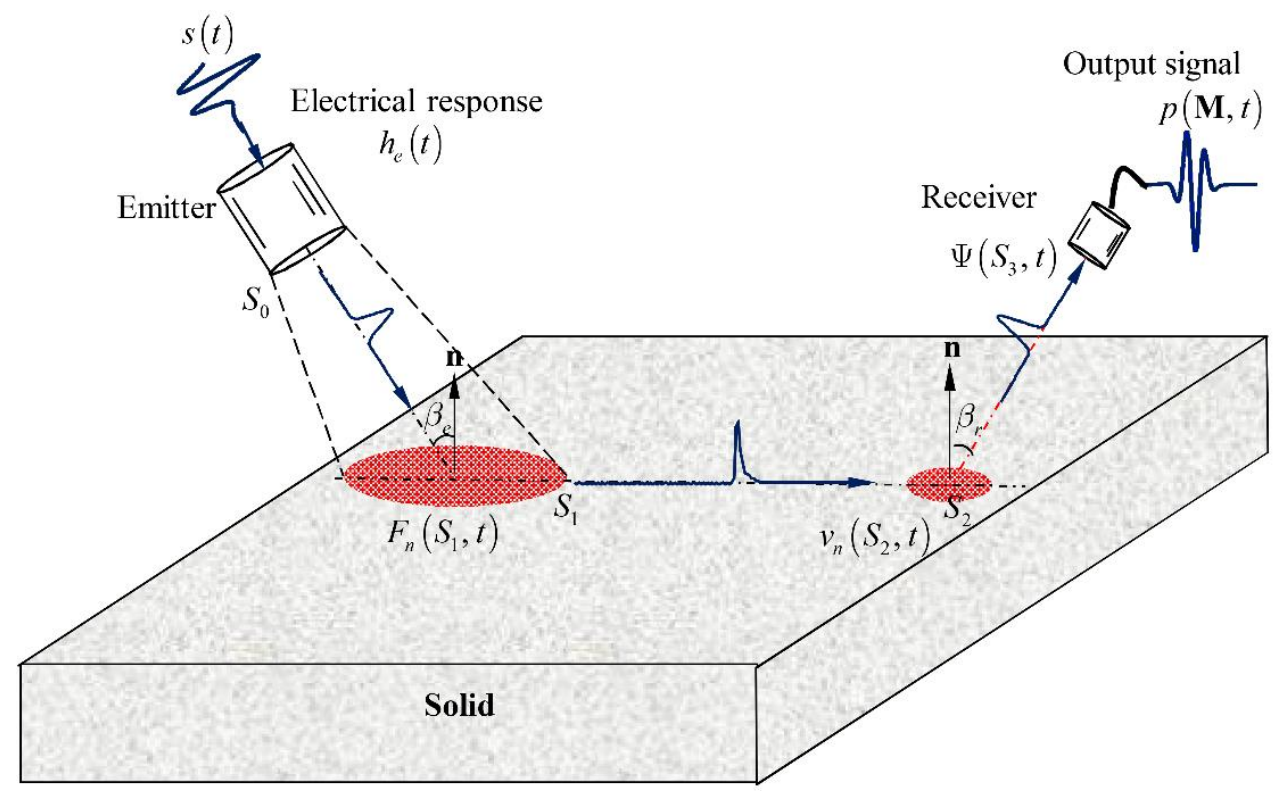

(a)

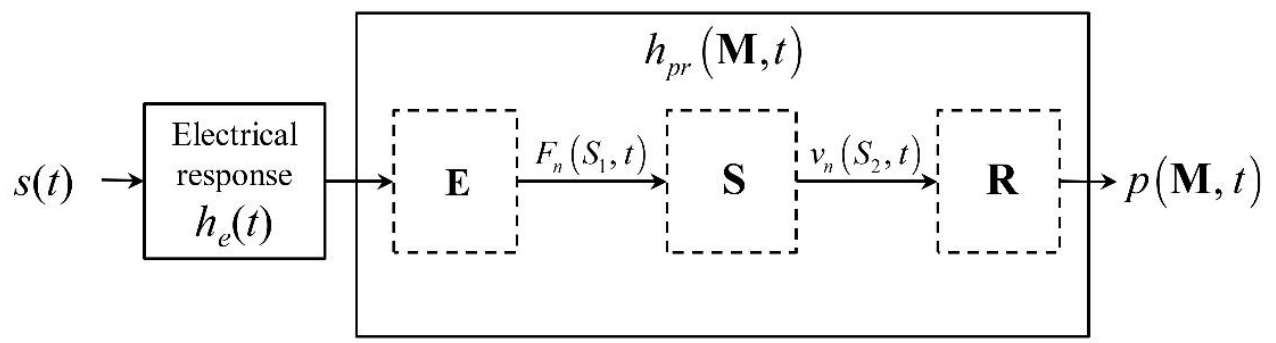

(b)

Fig.1 (a) Geometry and notation used for the ESR system model; (b) Equivalent block diagram of the computational procedure.

- Step 3 describes the SW re-radiation from sample surface $S_{2}$ to the air-coupled receiver $S_{3}$, and is characterized by block $\mathbf{R}$.

\subsection{Step 1: Acoustic wave propagation from air-coupled emitter to solid sample}


The notations and geometry used for the modeling of Step 1 are presented in Fig. 2. An ultrasonic wave is radiated from the emitter surface $S_{0}$ into the air, then impinges on the sample surface $S_{1}$ and produces a vertical force $F_{n}\left(\mathbf{M}_{1}, t\right)$. The aim of step 1 is to model $F_{n}\left(\mathbf{M}_{1}, t\right)$ over the surface $S_{1}$.

According to the solution presented in $R E F$ (Eq. (10)), and using the notations defined in Fig.2, the pulse response in terms of pressure, representing the field incident on the sample surface $S_{1}$ radiated from emitter $S_{0}$, which is excited by a normal component velocity in the form of a Dirac function $v_{n}\left(\mathbf{M}_{0}, t\right)=v_{n}\left(\mathbf{M}_{0}\right) \delta(t)$ (assuming piston mode operation of the emitter), and observed at point $\mathbf{M}_{1}$, can be written as:

$$
h_{p}\left(\mathbf{M}_{1}, t\right)=\rho \frac{\mathrm{d}}{\mathrm{d} t} \int_{S_{0}} v_{n}\left(\mathbf{M}_{0}\right) g_{\alpha_{f}}\left(\mathbf{M}_{0}, \mathbf{M}_{1}, \alpha_{f}, t\right) \mathrm{d} S_{0}
$$

where $g_{\alpha_{f}}\left(\mathbf{M}_{0}, \mathbf{M}_{1}, \alpha_{f}, t\right)$ is the Green's function in absorbing air, $\mathbf{M}_{0}, \mathbf{M}_{1}$ are points on the surfaces $S_{0}, S_{1}$, respectively, and $\rho$ is the density of air. The attenuation in air, $\alpha_{f}$, obeys the power law:

$$
\alpha_{f}(\omega)_{\mathrm{dB} / \mathrm{cm}}=\alpha_{f 0} \omega^{\eta}
$$

Various studies $[44,45]$ have confirmed that the attenuation in air can be expressed as a quadratic function of frequency, i.e. $\eta=2$, with $\alpha_{f 0}=\alpha_{1} \sqrt{T / T_{0}} /\left(4 \pi^{2} P / P_{0}\right)$ and $\alpha_{1}=15.895 \times 10^{-11}\left[\mathrm{~dB} /\left(\mathrm{m} \cdot \mathrm{Hz}^{2}\right)\right]$, where $T$ is the temperature, $P$ is the pressure, and the

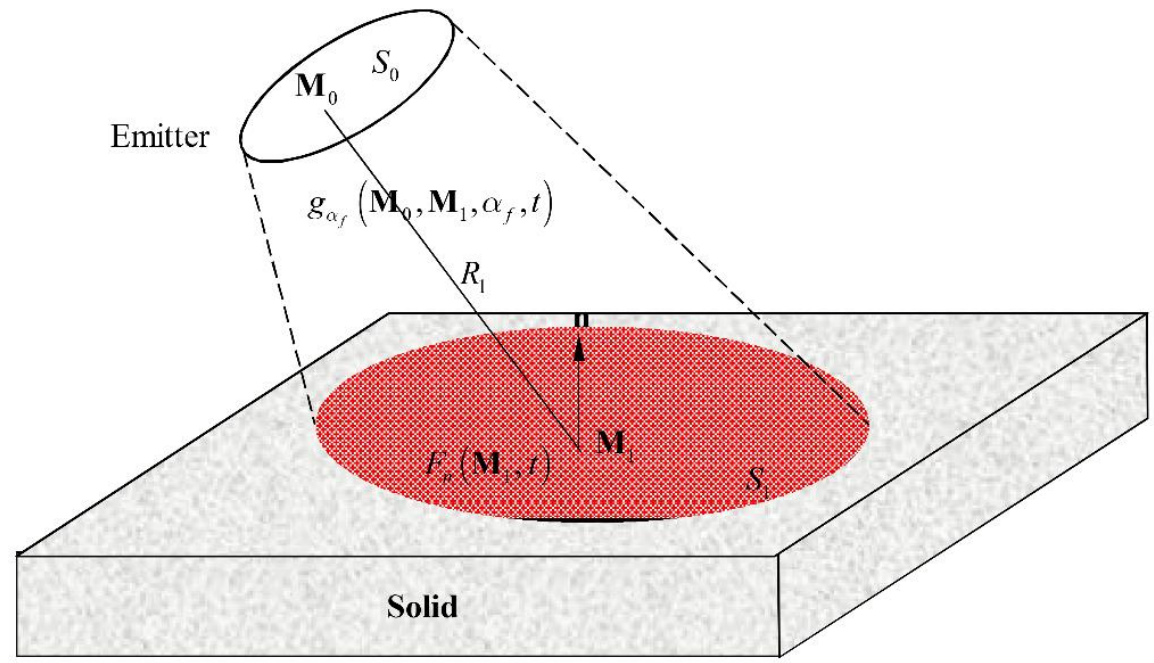

Fig. 2 Notations for step 1 
reference values are $T_{0}=25^{\circ} \mathrm{C}$ and $P_{0}=101.325 \mathrm{kPa}$, respectively (for these calculations $\alpha$ must be expressed in units of $1 / \mathrm{m}$, where: $\left.\alpha_{\mathrm{dB} / \mathrm{m}}=\alpha_{1 / \mathrm{m}} \times 20 \log _{10}(e)=8.68 \alpha_{1 / \mathrm{m}}\right)$. As shown in $R E F$, for the special case where $\eta=2$ the attenuation-induced phase shift is zero, the medium is dispersion free, and the phase velocity in air is constant: $c(\omega)=c(\infty)=c_{f}$. The causal Green's function for air in the frequency domain can then be expressed as (see REF; Eq. (9)):

$$
G_{\alpha_{f}}\left(\mathbf{M}_{0}, \mathbf{M}_{1}, \alpha_{f}, \omega\right)=\frac{e^{-\alpha_{f 0} \omega^{2} R_{1}+j\left(\frac{\omega}{c_{f}} R_{1}-\omega t\right)}}{2 \pi R_{1}}
$$

where $R_{1}=\left|\mathbf{M}_{1}-\mathbf{M}_{0}\right|$. The Green's function $g_{\alpha_{f}}$ for air in the time domain can then be obtained using the inverse Fourier transform, as (see REF; Eq. (4)):

$$
g_{\alpha_{f}}\left(\mathbf{M}_{0}, \mathbf{M}_{1}, \alpha_{f}, t\right)=\mathcal{F}^{-1}\left[G_{\alpha_{f}}\left(\mathbf{M}_{0}, \mathbf{M}_{1}, \alpha_{f}, \omega\right)\right]
$$

As $G_{\alpha_{f}}$ in Eq. (3) has a Gaussian shape, the function $g_{\alpha_{f}}$ for air can also be determined analytically (REF; Eq. (12)). In computational terms, this solution is more efficient.

The acoustic wave passes through the air and "impinges on" the point $\mathbf{M}_{1}$ on the solid surface with a force $F\left(\mathbf{M}_{1}, t\right)$, which excites a SW in the solid medium. The normal force $F_{n}\left(\mathbf{M}_{1}, t\right)$, expressed in units of force/unit area, can be derived from the pressure gradient of the incident wave [46] as:

$$
F_{n}\left(\mathbf{M}_{1}, t\right)=\frac{\partial p\left(\mathbf{M}_{1}, t\right)}{\partial n}
$$

By using the known relationship between pressure and velocity potential: $h_{p}\left(\mathbf{M}_{1}, t\right)=\rho \frac{\mathrm{d} h_{\Psi}\left(\mathbf{M}_{1}, t\right)}{\mathrm{d} t}$, the force distribution over the surface $S_{1}$ can be expressed as:

$$
F_{n}\left(\mathbf{M}_{1}, t\right)=\rho \frac{\mathrm{d}}{\mathrm{d} t} \frac{\partial h_{\Psi}\left(\mathbf{M}_{1}, t\right)}{\partial n}
$$

In fact, the above equations state that the local force acting on the mass of the local sample is proportional to its acceleration (second Newton's law). Using Eq.(1), we thus obtain:

$$
F_{n}\left(\mathbf{M}_{1}, t\right)=\rho \frac{\mathrm{d}}{\mathrm{d} t}\left[\int_{S_{0}} v_{n}\left(\mathbf{M}_{0}\right) \frac{\partial}{\partial n} g_{\alpha_{f}}\left(R_{1}, \alpha_{f}, t\right) \mathrm{d} S_{0}\right]
$$

where $n$ is the normal vector. The normal derivative can be written as:

$$
\frac{\partial}{\partial n} g_{\alpha_{f}}\left(R_{1}, \alpha_{f}, t\right)=\frac{\partial g_{\alpha_{f}}\left(R_{1}, \alpha_{f}, t\right)}{\partial R_{1}} \cdot \frac{\partial R_{1}}{\partial n}=\frac{\partial g_{\alpha_{f}}\left(R_{1}, \alpha_{f}, t\right)}{\partial R_{1}} \cos \left\langle R_{1}, n\right\rangle
$$


where $\left\langle R_{1}, n\right\rangle$ is the angle between the incident wave and the normal vector $n$. Since in the modeled system we have $R_{1} \square \lambda_{f}$ (distance from emitter to sample $R_{1 \min } \approx 30 \mathrm{~mm}$ and $\lambda_{\text {fmax }}=3 \mathrm{~mm}$, see Section.3), the so-called plane wave approximation $\frac{\mathrm{d}}{\mathrm{d} R} \approx-\frac{1}{c} \frac{1}{\mathrm{~d} t}$ [47] can be used. Eq. (7) can then be simplified to:

$$
F_{n}\left(\mathbf{M}_{1}, t\right)=-\rho \frac{1}{c} \frac{\mathrm{d}^{2}}{\mathrm{~d} t^{2}}\left[\int_{S_{0}} v_{n}\left(\mathbf{M}_{0}\right) g_{\alpha_{f}}\left(R_{1}, \alpha_{f}, t\right) \cos \left\langle R_{1}, n\right\rangle \mathrm{d} S_{0}\right]
$$

At a plane interface, the fluid air and solid are characterized by the acoustic impedances $Z_{f}$ and $Z_{s}$, respectively. As these two impedances are different, reflection and transmission of the incident wave, is characterized by [48]:

$$
\gamma_{t}=\frac{2 Z_{f}}{Z_{f}+Z_{s}}, \gamma_{r}=\frac{Z_{s}-Z_{f}}{Z_{f}+Z_{s}}
$$

where $\gamma_{t}$ and $\gamma_{r}$ are the transmission and reflection coefficients in terms of pressure, for normal incidence, and the subscripts $r$ and $t$ denote reflected and transmitted waves, respectively. Since pressure is proportional to the local force, the distribution of the effective normal force $F_{n e}\left(\mathbf{M}_{1}, t\right)$ over $S_{1}$ becomes:

$$
F_{n e}\left(\mathbf{M}_{1}, t\right)=\gamma_{t} F_{n}\left(\mathbf{M}_{1}, t\right)
$$

It should be noted that we always have $Z_{s} \square Z_{f}$, such that $\gamma_{t} \square 1$, which means that in an air-coupled system only a small fraction of the energy in the incident wave penetrates the solid [49]. Finally, the field of $F_{n e}\left(\mathbf{M}_{1}, t\right)$ over the surface $S_{1}$ can be written as:

$$
F_{n e}\left(\mathbf{M}_{1}, t\right)=-\gamma_{t} \rho \frac{1}{c} \frac{\mathrm{d}^{2}}{\mathrm{~d} t^{2}}\left[\int_{S_{0}} v_{n}\left(\mathbf{M}_{0}\right) g_{\alpha_{f}}\left(R_{1}, \alpha_{f}, t\right) \cos \left\langle R_{1}, n\right\rangle \mathrm{d} S_{0}\right]
$$

\subsection{Step 2: Surface wave propagation along the sample}

The notation for the modeling of Step 2 is presented in Fig.3. In a solid medium, the vertical point load $F_{n e}\left(\mathbf{M}_{1}, t\right)$ excites both body waves and SWs, which propagate along its surface. This topic has been extensively studied and is known as Lamb's problem [50]. The SWs always dominate, because $2 / 3$ of the energy is converted into SWs, whilst the remainder is converted to body waves [51]. Measurements performed by Breckenridge and Greenspan [52] proved that the SW Green's function in a lossless solid could be approximated by a Dirac delta function: 


$$
g\left(\mathbf{M}_{1}, \mathbf{M}_{2}, t\right)=\frac{1}{2 \pi \sqrt{R_{2}}} \delta\left(t-\frac{R_{2}}{c_{R}}\right)
$$

where $R_{2}=\left|\mathbf{M}_{2}-\mathbf{M}_{1}\right|$, and $c_{R}$ is the SW velocity. Using the solution for body waves given by Aki and Richards [53], the local normal impulse load $F_{n e}\left(\mathbf{M}_{1}\right) \delta(t) \mathrm{d} S_{1}$ excites SWs, and for a lossless solid the SW impulse response in terms of displacement can be approximated by:

$$
h_{u_{n}}\left(\mathbf{M}_{2}, t\right)=\int_{S_{1}} F_{n e}\left(\mathbf{M}_{1}\right) \frac{1}{2 \pi \sqrt{R_{2}}} \delta\left(t-\frac{R_{2}}{c_{R}}\right) \mathrm{d} S_{1}
$$

where the term $1 /\left(2 \pi \sqrt{R_{2}}\right)$ represents the geometrical spreading of a cylindrical wave [54]. A similar formula has also been used to compute the longitudinal and transversal field in solid samples since, as mentioned above, any load $F_{n}$ applied to the surface of a solid generates all types of wave [55]. The above formula states that when an excitation force $F_{n e}\left(\mathbf{M}_{1}\right) \delta(t)$ is exerted on a unit area $\mathrm{d} S_{1}$, a surface wave is produced, which propagates along the outer surface of the solid, reaches point $\mathbf{M}_{2}$ on $S_{2}$, and produces a displacement response $h_{u}\left(\mathbf{M}_{2}, t\right)$. It should be pointed out that the SW includes both longitudinal and normal components, which are shifted in phase with respect to each other by $\pi / 2$. The ratio between these components remains fixed and depends only on Poisson's ratio in the solid [12]. Since in step 3 the leaky wave is radiated due to the normal component of the SW, we assume that the integral expressed in (14) determinates the vertical displacement. In the present case, since the force distribution $F_{n e}\left(\mathbf{M}_{1}, t\right)$ over $S_{1}$ is not separated in time and space, Eq. (14) can be rewritten as:

$$
u_{n}\left(\mathbf{M}_{2}, t\right)=\int_{S_{1}} F_{n e}\left(\mathbf{M}_{1}, t\right) * \frac{1}{2 \pi \sqrt{R_{2}}} \delta\left(t-\frac{R_{2}}{c_{R}}\right) \mathrm{d} S_{1}
$$

where * denotes a time-domain convolution, and $u_{n}$ is the normal component of the

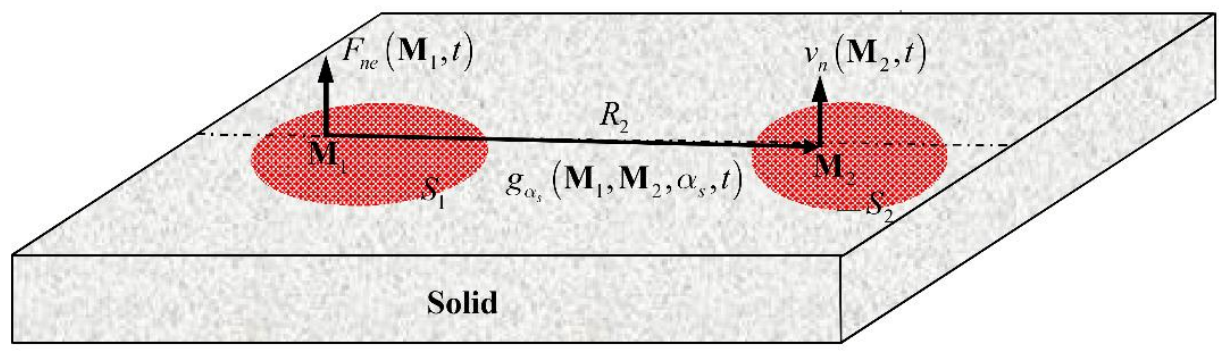

Fig.3 Notations for computational step 2: SW propagation 
displacement distribution over surface $S_{2}$.

Equations (14) and (15) provide an approximate description of the SW field. The physical meaning of Eq. (14) is that every point may be considered as the source of an outgoing wavelet, such that the field at any arbitrary point can be constructed from the superposition of these wavelets. This is equivalent to Huygens' principle, which is used by Wilcox et al. [56] to model the Lamb wave field excited by PVDF IDT. This idea was also adopted by Schmerr-Jr. and Sedov [57]. A similar technique was used to model the SW field generated by an angle beam wedge transducer [58, 59] and in the case of transducer arrays for Lamb wave generation,[57,58] Recently, it was successfully used to model the acoustic field of Inter Digital Transducers (IDT), and was verified experimentally [59].

If absorption in the solid is taken into account, the integral in (15) can be written in the form:

$$
u_{n}\left(\mathbf{M}_{2}, t\right)=\int_{S_{1}} F_{n e}\left(\mathbf{M}_{1}, t\right) * g_{\alpha_{s}}\left(\mathbf{M}_{1}, \mathbf{M}_{2}, \alpha_{s}, t\right) \mathrm{d} S_{1}
$$

where $g_{\alpha_{s}}\left(\mathbf{M}_{1}, \mathbf{M}_{2}, \alpha_{s}, t\right)$ is the SW Green's function in a lossy solid medium, characterized by the attenuation $\alpha_{s}$. In general, the solid may have a frequency-dependent attenuation of the form:

$$
\alpha_{s}(\omega)=\alpha_{s 1} \omega^{\eta}+\alpha_{s 0}
$$

Similarly, as in Section (2.1), the velocity dispersion $c(\omega)$ should be introduced in order to preserve the causality of the function $g_{\alpha_{s}}$. Since the attenuation in most solids [60], including the solid used in the present study, varies linearly as a function of frequency, we introduce the dispersion relationship proposed by Waters, Kelly and McGough et al. [61-65], which is applicable to the case where $\eta=1$ :

$$
\frac{1}{c(\omega)}-\frac{1}{c\left(\omega_{0}\right)}=-\frac{2}{\pi} \alpha_{s 1} \ln \left|\frac{\omega}{\omega_{0}}\right|
$$

where $c_{\infty}=c_{R}$ is the limiting value of $c(\omega)$ when $\omega \rightarrow \infty$ (corresponding to the $c_{R}$ velocity of a SW in a lossless medium), and $\omega_{0}$ is a constant, which should be significantly greater than the maximum frequency of interest, i.e. $\omega_{0} \square \omega$. The "causal" Green's function for the case where $\eta=1$ can then be written as (cf. Eqs. (3) and (13)): 


$$
G_{\alpha_{s}}\left(\mathbf{M}_{1}, \mathbf{M}_{2}, \alpha_{s}, \omega\right)=\frac{e^{-\alpha_{s 0} R_{2}-\alpha_{s 1} \omega R_{2}+j\left(-\frac{2 \alpha_{s 1} \omega}{\pi} \ln \left|\frac{\omega}{\omega_{0}}\right| R_{2}+\frac{\omega}{c_{R}} R_{2}-\omega t\right)}}{2 \pi \sqrt{R_{2}}}
$$

(If $\eta \neq 1$ the solutions adapted to cases where $0<\eta \leq 2, \eta \neq 1$, already described in Eq.(3), can be used). The above expression is the counterpart of Eq.(3), in which the geometrical spreading of a cylindrical wave is introduced. The causal Green's function of the SW in the time domain, $g_{\alpha_{s}}\left(\mathbf{M}_{1}, \mathbf{M}_{2}, \alpha_{s}, t\right)$, can then be obtained using the inverse Fourier transform, as in Eq.(4).

Finally, by taking $v_{n}=\mathrm{d} u_{n} / \mathrm{d} t$, the normal component of the velocity $v_{n}$ over $S_{2}$ becomes:

$$
v_{n}\left(\mathbf{M}_{2}, t\right)=\frac{\mathrm{d}}{\mathrm{d} t} \int_{S_{1}} F_{n e}\left(\mathbf{M}_{1}, t\right) * g_{\alpha_{s}}\left(\mathbf{M}_{1}, \mathbf{M}_{2}, \alpha_{s}, t\right) \mathrm{d} S_{1}
$$

\subsection{Step 3: Surface wave re-radiation from the structure's surface to an air-coupled receiver}

In step 3, the surface $S_{2}$ of the solid sample acts as an emitter of a so-called leaky wave [66]. In practice, re-radiation of a leaky wave from a solid surface into air corresponds to the problem of radiation from a rigid, baffled emitter, which is already accounted for in Step 1. Using the notations provided in Fig. 4, the pulse response expressed in terms of the velocity potential observed at point $\mathbf{M}_{3}$ on the receiver, in response to radiation from $S_{2}$ excited by $v_{n}\left(\mathbf{M}_{2}\right) \delta(t)$, can be written as:

$$
h_{\Psi}\left(\mathbf{M}_{3}, t\right)=\gamma_{t} \int_{S_{2}} v_{n}\left(\mathbf{M}_{2}\right) * g_{\alpha_{f}}\left(\mathbf{M}_{2}, \mathbf{M}_{3}, \alpha_{f}, t\right) \mathrm{d} S_{2}
$$

By analogy with Eq. (15), the velocity potential over the receiver surface $S_{3}$ can be expressed as:

$$
\Psi\left(\mathbf{M}_{3}, t\right)=h_{\Psi}\left(M_{3}, t\right) * v_{n}\left(\mathbf{M}_{2}, t\right)=\gamma_{t} \int_{S_{2}} v_{n}\left(\mathbf{M}_{2}, t\right) * g_{\alpha_{f}}\left(\mathbf{M}_{2}, \mathbf{M}_{3}, \alpha_{f}, t\right) \mathrm{d} S_{2}
$$

Similar to the approach used in $R E F$, the response at the receiver output is found by computing the spatial average of $\Psi\left(\mathbf{M}_{3}, t\right)$ over the surface $S_{3}$, and converting the potential into pressure. Finally, the output pressure response of the receiver centered at point $\mathbf{M}$ can be written as: 


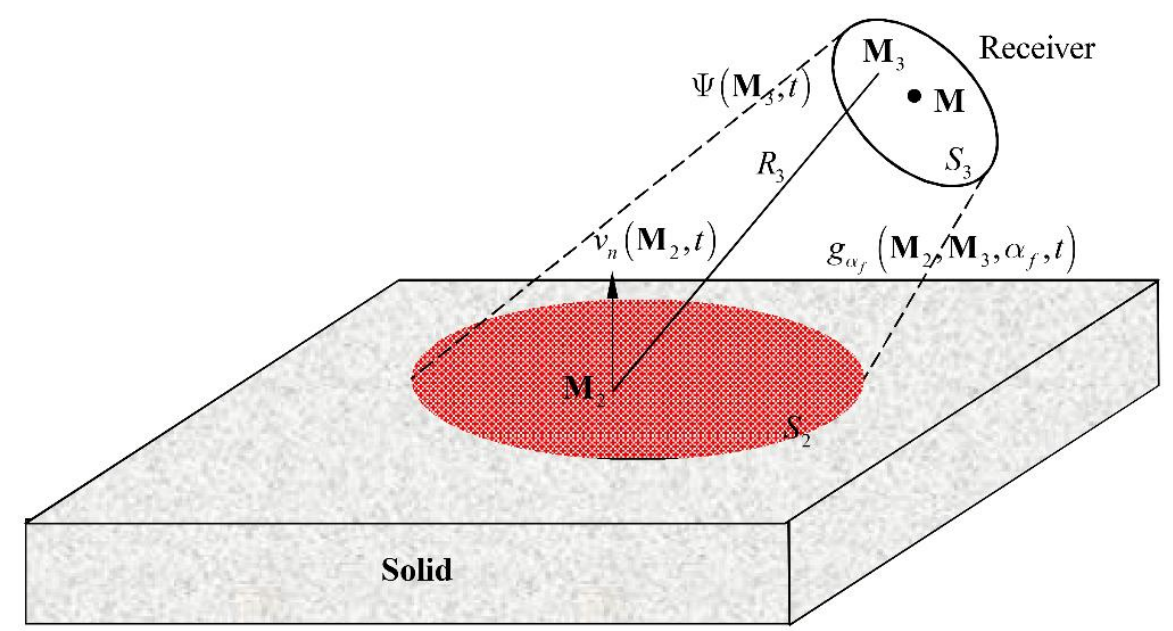

Fig.4 Notations for step 3- receiver, centered on the point $\mathbf{M}$

$$
\begin{aligned}
h_{p r}(\mathbf{M}, t) & =\rho \frac{\mathrm{d}}{\mathrm{d} t} \frac{1}{S_{3}} \int_{S_{3}} \Psi\left(\mathbf{M}_{3}, t\right) \mathrm{d} S_{3} \\
& =\gamma_{t} \rho \frac{\mathrm{d}}{\mathrm{d} t} \frac{1}{S_{3}} \int_{S_{3}}\left[\int_{S_{2}} v_{n 2}\left(\mathbf{M}_{2}, t\right) * g_{\alpha_{f}}\left(\mathbf{M}_{2}, \mathbf{M}_{3}, \alpha_{f}, t\right) \mathrm{d} S_{2}\right] \mathrm{d} S_{3}
\end{aligned}
$$

It is interesting to note that for the SW propagating across $S_{2}$, the field $v_{n}\left(\mathbf{M}_{2}, t\right)$ over $S_{2}$ acts as a time delay beam-former, such that the $\mathrm{SW}$ is naturally re-radiated from the sample's surface towards the receiver, at an inclination angle $[12,67]$ :

$$
\beta_{o}=\sin ^{-1}\left(c_{f} / c_{R}\right)
$$

The inclination of the receiver $\beta_{r}$ is thus set to $\beta_{r}=\beta_{o}$, such that waves observed at $S_{3}$ arrive in phase, and their superposition provides a signal maximum at the receiver output. Similarly, if the inclination $\beta_{e}$ of the emitter (Fig.2) is set to $\beta_{e}=\beta_{o}$, this provides optimal SW excitation [17] (see also Appendix B).

\subsection{Total system response observed at the receiver output}

In accordance with the setup shown in Fig.1, in the presence of an excitation $s(t)=\delta(t)$ the impulse response of the entire ESR system can be written as:

$$
h_{p E S R}(\mathbf{M}, t)=\delta(t) * h_{e}(t) * h_{p r}(\mathbf{M}, t)=h_{e}(t) * h_{p r}(\mathbf{M}, t)
$$

where $h_{e}(t)$ is the electric response characterizing the electric-acoustic properties of the emitter-receiver pair, defined and used in REF. Note that the response $h_{p r}$ cannot be determined from the simple convolution of the responses of consecutive blocks $\mathbf{E}, \mathbf{S}, \mathbf{R}$ in Fig.1, because the blocks $\mathbf{S}$ and $\mathbf{R}$ contain the spatio-temporal input signal, whereas the integrals over $S_{1}, S_{2}$, and $S_{3}$ are not space invariant. 


\section{Experimental system}

In order to validate the theoretical models developed in section 2 , the theoretically predicted field was compared with experimental measurements. The emitter was held in a constant position and the receiver was mounted on an accurate 3D scanner, allowing the signal to be recorded along the $x, y$, and $z$ axes (Fig. 5).

A circular, piezoelectric air-coupled transducer (Ultran Group USA[68]), with an active diameter $D_{E}=50 \mathrm{~mm}$ and central frequency $f_{o}=350 \mathrm{kHz}$, was used as the emitter. A membrane type, non-contact, wide-band $(10 \mathrm{kHz}-2 \mathrm{MHz})$ receiver (type mBAT-1, Micro-Acoustic Canada [69]) with a membrane diameter $D_{R}=10 \mathrm{~mm}$ was used for signal reception (the receiver and emitter were the same as those characterized in $R E F$ ). The solid sample used in the experiment was a $600 \mathrm{~mm} \times 400 \mathrm{~mm} \times 48 \mathrm{~mm}$ rectangular block of Plexiglas (poly(methyl methacrylate)). Its thickness $d=48 \mathrm{~mm}$ was chosen to be much greater than the maximum wavelength of the SW, in order to avoid the production of Lamb waves. The sheet of sponge foam used in this setup provides insulation from direct sound waves (transmitted directly through the air, from the emitter to the receiver).

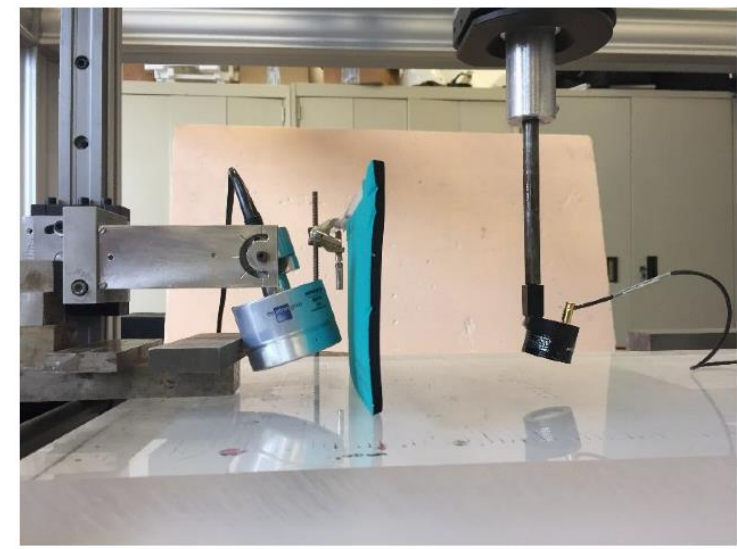

(a)

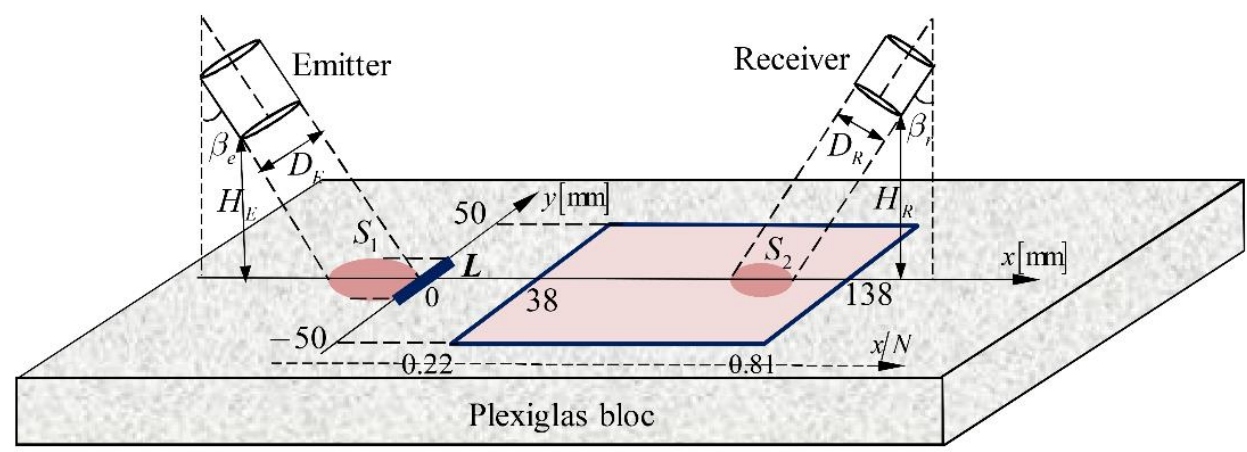

(b)

Fig.5 (a) Experimental setup (b) Geometry and notation used for the experiments and computations 
The user-defined model parameters are: emitter and receiver heights $H_{E}, H_{R}$ and inclination angles of the emitter and receiver $\beta_{e}, \beta_{r}$ (Fig. 5(b)). The experimental velocity of sound in air and Plexiglas were determined, as described in Appendix A. Using the data provided in Table 2 together with Eq. (24), the optimal inclinations $\beta_{e}$ and $\beta_{r}$ were determined as: $\beta_{o}=\sin ^{-1}(345 / 1270)=15.8^{\circ}$. This value was confirmed experimentally, by measuring the received signal amplitude as a function of $\beta_{e}$ and $\beta_{r}$ : the strongest signal level was indeed found for $\beta_{e}=\beta_{r}=15.7^{\circ} \pm 0.2^{\circ}$, and this angle was then used for all experiments and calculations. The user-defined geometrical parameters used during the experiments are summarized in Table 1.

Table 1 Geometric parameters of the experiment

\begin{tabular}{|c|c|c|c|c|}
\hline & $\begin{array}{c}\text { Diameter } \\
{[\mathrm{mm}]}\end{array}$ & $\begin{array}{c}\text { Height } \\
{[\mathrm{mm}]}\end{array}$ & $\begin{array}{c}\text { Inclination } \\
(\mathrm{deg})\end{array}$ & $\begin{array}{c}\text { Scanned area } \\
{[\mathrm{mm}]}\end{array}$ \\
\hline Emitter $S_{0}$ & $D_{E}=50$ & $H_{E}=15.9$ & $\beta_{e}=15.8^{\circ}$ & \multirow{2}{*}{$\begin{array}{c}38<x<138 \\
-50<y<50\end{array}$} \\
\hline Receiver $S_{3}$ & $D_{R}=10$ & $H_{R}=23.3$ & $\beta_{r}=15.8^{\circ}$ & -50 \\
\hline
\end{tabular}

Measurements of the impulse response $h_{P E S R}$ were made over the $38<x<138 \mathrm{~mm}$, $-50<y<50 \mathrm{~mm}$ region. In Appendix B we show that the line $\boldsymbol{L}$ (Fig. 5(b)) can be interpreted as being equivalent to a SW source, which replaces $S_{1}$. The position of $\boldsymbol{L}$ was thus assumed to be $x=0$, allowing the far-field limit $N$ of this equivalent source to be estimated as [70]:

$$
N=L^{2} /\left(4 \lambda_{o R}\right)=173 \mathrm{~mm}
$$

where $\lambda_{o R}=c_{R}\left(f=f_{o}\right) / f_{o}=3.6 \mathrm{~mm}$ is the $\mathrm{SW}$ wavelength in Plexiglas at the central frequency $f_{o}$. The studied zone is thus located inside the near-field region: $0.21 \leq x / N \leq 0.81$

As a consequence of the very low transmission coefficient $\gamma_{t}$ between air and the solid, the same chirp technique as that described in $R E F$ was applied in this experiment, to improve the signal to noise ratio $S N R$ of the observed signals $[10,11,43]$. The linear chirp signal $\operatorname{ch}(t)$, covering a bandwidth $B_{c h}$ from $5-700 \mathrm{kHz}$, was the same as that used in $R E F$. The chirp duration was adjusted to $80 \mathrm{~ms}$, the longest duration compatible with the processor memory used during the measurements. The resulting improvement in SNR (compared to the level obtained using a pulsed excitation) was approximately 2.5 times greater than that obtained in $R E F$, and is estimated to be $25 \mathrm{~dB}$. The chirp signal is amplified and excites the 
emitter with an amplitude of $70 \mathrm{~V}$, in order to avoid thermal damage to the emitter.

From Eq. (25), if $s(t)=\operatorname{ch}(t)$ the receiver provides the non-correlated output signal:

$$
p_{n c}(\mathbf{M}, t)=\operatorname{ch}(t) * h_{p E S R}(\mathbf{M}, t)
$$

This signal is then compressed by the next correlation with $\operatorname{ch}(t)$ :

$$
p_{c o r r}(\mathbf{M}, t)=\operatorname{ch}(t) \otimes\left[\operatorname{ch}(t) * h_{p E S R}(\mathbf{M}, t)\right]=k(t) * h_{p E S R}(\mathbf{M}, t)
$$

where $k(t)=\operatorname{ch}(t) \otimes \operatorname{ch}(t)$ is the compressed chirp (known as a Klauder wavelet, [71]). It has been shown in $R E F$ that under these experimental conditions, the linear chirp with a

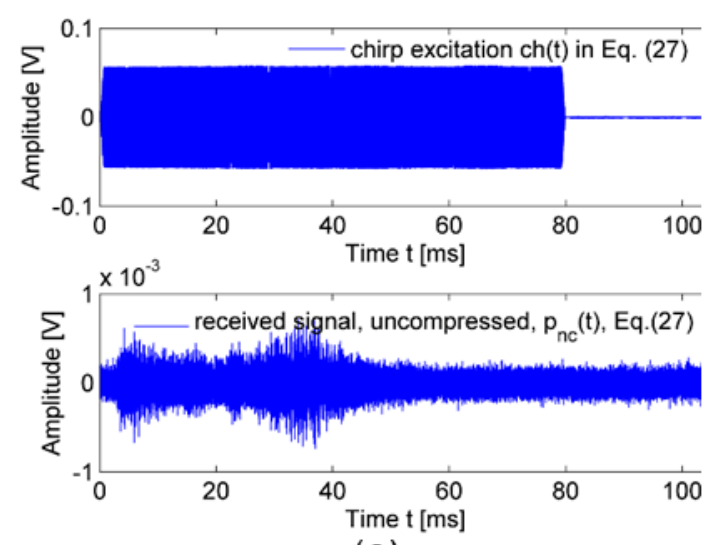

(a)

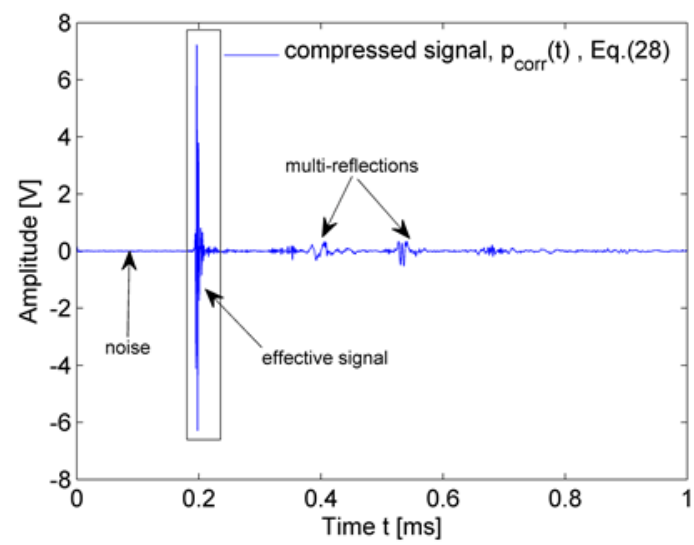

(b)

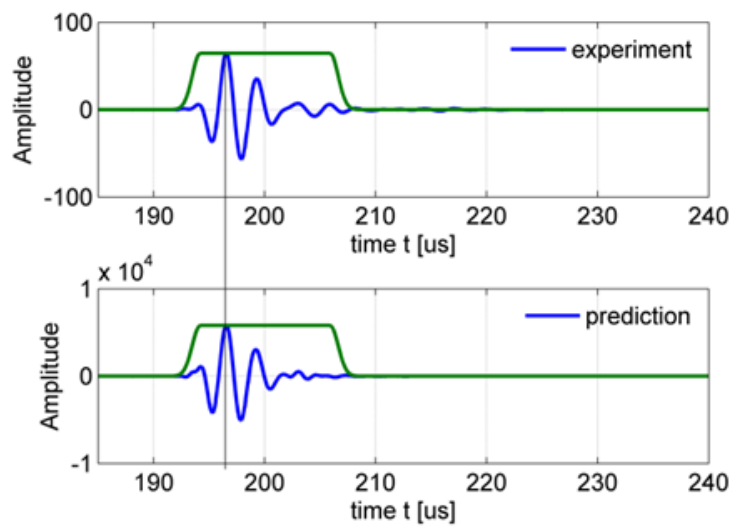

(c)

Fig.6 (a) The upper portion of this figure shows the applied chirp signal $\operatorname{ch}(t)$ of duration $T_{c h}=80 \mathrm{~ms}$ and bandwidth $5-700 \mathrm{kHz}$; the lower portion shows the uncompressed received signal $p_{n c}$ (Eq.(27)) recorded at the point $(x=66 \mathrm{~mm}, y=0)$; (b) The signal after compression $p_{\text {corr }}$, derived from Eq. (28). The response of interest, $h_{p E S R}$ corresponds to the first arrival of $p_{c o r r}$; (c) Upper portion: windowed first arrival of the experimental signal $p_{\text {corr }}$ shifted in order to match the peak of the predicted signal; Lower portion: predicted signal inside the same time-domain window. 
5-700 kHz bandwidth acts like a Dirac delta function. Eq. (28) can thus be simplified to:

$$
p_{\text {corr }}(\mathbf{M}, t) \approx \delta(t) * h_{p E P R}(\mathbf{M}, t)=h_{p E P R}(\mathbf{M}, t)
$$

The chirp signal $c h(t)$, the received signal $p_{n c}(t)$, and the compressed signal $p_{c o r r}$ are shown in Figs. $6(\mathrm{a}, \mathrm{b})$. The response of interest, $h_{p E S R}$, corresponds to the first arrival of $p_{\text {corr }}$ and is followed by different diffracted components of the air coupled waves. Using the same approach as described in $R E F$, this response is thus extracted through the use of an automatically defined window, as shown in Fig. 6(c). In the present study, the length of this window is defined as the interval over which the normalized predicted signal is greater than $10^{-2}$, ( i.e. corresponds to $S N R=20 \mathrm{~dB}$ ).

It should be noted that generally dispersion causes signals change shape as a function of propagation distance and limits the effectiveness of the chirp technique [72]. In the presented study the Rayleigh wave was intrinsically non-dispersive and the air was not dispersive either. The only dispersion was induced by the Plexiglas absorption (Eq .(A.7)); it was very small (less than $0.1 \%$, Fig. A.4) and thus did not perturb the chirp technique. If the intrinsically dispersive waves were processed (e.g. the Lamb and Love SH waves ) the chirp technique could be used, but under specific requirements [73].

\section{Experimental validation and model prediction errors}

\subsection{Prediction}

The response $h_{p E S R}(\mathbf{M}, t)$ of the modeled ESR system is computed by assuming the excitation signal to be $s(t)=\delta(t)$, and by sequentially computing Eqs. (12), (20), (23) and (25). The electric response of the emitter-receiver pair $h_{e}(t)$ is the same as that determined experimentally, which was also used in $R E F$. The results obtained with this method are referred to as the "exact model". Independently $h_{P E S R}(\mathbf{M}, t)$ is predicted by the simplified model, proposed in Appendix B.

The solution for the response $h_{p r}(t)$ was determined numerically, using the same Discrete Representation (DR) computational technique as that described in REF [74]. Although a DR model is included in the DREAM toolbox software environment [75], it was preferred to develop a DR routine independently under the Matlab environment, in order to include variable power law attenuation in the model, with $\eta=2$, as required in steps 1 and 3 
(DREAM currently only includes the case where $\eta=1$ ). The computations were performed at a sampling frequency $f_{s}=10 \mathrm{MHz}$ (to ensure that the condition $f_{s} \square f_{\max }$ - where $f_{\max }$ is the highest frequency of interest - is adequately respected), and used spatial discretization of the surfaces $S_{0}, S_{1}, S_{2}, S_{3}$ defined by $\Delta x, \Delta y=1 \mathrm{~mm}$. With the above settings, it was verified that the computational errors did not exceed $0.1 \%$.

The input geometrical parameters were the same as those used for the experiment (Table 1). Although the relevant data can be found in the bibliography, the velocity and attenuation of Plexiglas were determined experimentally, in order to ensure the best possible predictive accuracy. The parameters used to compute the modeled acoustic field of the scanner are summarized in Table 2. Surfaces $S_{1}$ and $S_{2}$, were approximated as the projections of emitter $S_{0}$ and receiver $S_{3}$ onto the surface of the Plexiglas solid. Since the emitter and receiver were located very close to the solid sample, they were considered to be circular (they are in fact slightly elliptical $[23,24])$.

Table 2. Material properties used for the computations (Appendix A)

\begin{tabular}{|c|c|c|c|c|c|}
\hline Medium & Velocity & Attenuation & Dispersion & $\begin{array}{c}\text { Impedance } \\
{[76]} \\
\text { Rayl }\end{array}$ & $\begin{array}{c}\text { Density } \\
{[76]} \\
\mathrm{kg} / \mathrm{m}^{3}\end{array}$ \\
\hline Air & $\begin{array}{l}\text { Longitudinal wave } \\
c_{f}=345 \mathrm{~m} / \mathrm{s} \\
\left(\mathrm{T}=25^{\circ} \mathrm{C}\right) \\
\text { [77] see Appendix A }\end{array}$ & $\begin{array}{l}\alpha_{f}=\alpha_{f 0} f^{2} \mathrm{~dB} / \mathrm{cm} \\
\alpha_{f 0}=15.895 \times 10^{-11} \mathrm{~dB} /\left(\mathrm{m} \cdot \mathrm{Hz}^{2}\right) \\
\mathrm{T}=25^{\circ} \mathrm{C} \text {; see Eq. }(2) ;[44,45,78]\end{array}$ & No dispersion, (see $R E F$ ). & $z_{f}=414$ & $\rho_{f}=1.24$ \\
\hline Plexiglas & $\begin{array}{l}\text { Surface wave } \\
c_{R}(f=350 \mathrm{kHz})=1270 \\
\mathrm{~m} / \mathrm{s} \text {, see Appendix A } \\
\text { and [66] }\end{array}$ & $\begin{array}{l}\text { See Eq.(A.5) } \\
\begin{array}{l}\alpha_{s}=\alpha_{s 1} f+\alpha_{s 0} \quad \mathrm{~dB} / \mathrm{cm} \\
\alpha_{s 1}=3.41 \mathrm{~dB} /(\mathrm{cm} \cdot \mathrm{MHz}) \\
\alpha_{s 0}=0.13 \mathrm{~dB} / \mathrm{cm}\end{array}\end{array}$ & $\begin{array}{l}\text { See Eq. (18) } \\
\alpha_{s 1}=3.41 \mathrm{~dB} /(\mathrm{cm} \cdot \mathrm{MHz}) \\
\text { converted to } \mathrm{Np} . \\
f_{s}=10 \mathrm{MHz}, \\
\omega_{\infty}=100 \omega_{\max } ; f_{\max }=0.5 \mathrm{MHz} \\
c_{R}\left(\omega_{\infty}\right)=1300 \mathrm{~m} / \mathrm{s}\end{array}$ & $z_{s}=3.1 \times 10^{6}$ & $\rho_{s}=1150$ \\
\hline
\end{tabular}

\subsection{Prediction errors}

The predicted and measured acoustic fields were compared for three typical field characteristics, identical to those used in REF. Each of these was derived from both the time domain signal, and its spectrum. For the convenience of presentation, they are redefined in Table 3. 
Table 3. Field characteristics, derived from both the time domain signal and its spectrum, used to compare the predicted and measured acoustic fields

\begin{tabular}{|l|l|c|c|}
\hline No. & Field characteristic & Definition & Equation \\
\hline 1 & $\begin{array}{l}\text { Waveform impulse response } \\
h_{p E S R}(\mathbf{M}, t)\end{array}$ & $(29)$ \\
\hline 2 & Spectrum of $h_{p E S R}(\mathbf{M}, t)$ & $H_{p E S R}(\mathbf{M}, \omega)=\mid \mathcal{F}\left[h_{p E S R}(\mathbf{M}, t)\right]$ & $(30)$ \\
\hline 3 & $\begin{array}{l}\text { Peak value of } h_{p E S R}(\mathbf{M}, t), \text { found in } \\
\text { the time domain. }\end{array}$ & $p_{m}(x / N, y)=\left.\max \left[h_{p E S R}(x / N, y, t)\right]\right|_{t}$ & $(31)$ \\
\hline 4 & $\begin{array}{l}\text { Spectral amplitude for a fixed } \\
\text { frequency } f_{o}\end{array}$ & $H_{p E S R}\left(x / N, y, f=f_{o}\right)$ & $(32)$ \\
\hline 5 & $\begin{array}{l}\text { Directivity pattern at distance } x / N, \\
\text { derived from the time-domain signal. }\end{array}$ & $b(\theta, x / N)=\frac{p_{m}(\theta, x / N)}{\max \left[p_{m}(\theta, x / N)\right]}$ & $(33)$ \\
\hline 6 & $\begin{array}{l}\text { Directivity pattern at distance } x / N, \\
\text { for a fixed frequency } f_{o}\end{array}$ & $b\left(\theta, x / N, f=f_{0}\right)=\frac{H_{p E S R}\left(\theta, x / N, f=f_{0}\right)}{\max \left[H_{p E S R}\left(\theta, x / N, f=f_{0}\right)\right.}$ & $(34)$ \\
\hline
\end{tabular}

The prediction error is expressed in \% as the error energy normalized with respect to the measured energy:

$$
E=\frac{\sum_{i=1}^{N_{s}}\left[P_{m}(i)-P_{p}(i)\right]^{2}}{\sum_{i=1}^{N_{s}}\left[P_{m}(i)\right]^{2}} \times 100 \%
$$

This relative error was determined for each pair of measured and (modeled) predicted values, where $P_{m}$ and $P_{p}$ are vectors containing the (normalized) measured and predicted quantities defined for the above field characteristics, and $N_{s}$ is the length of these vectors [79].

The measured field characteristics are compared with the predictions. Fig.7 provides examples of the three measured and predicted field characteristics defined above. It can be seen that for all characteristics the prediction error is quite small, thus demonstrating the high accuracy of the computational procedure proposed in this study. The most demanding test is that involving a comparison of the waveforms (Fig. 7(a), field characteristic No. 1), which is shown to have an error of approximately $2 \%$ for the exact model. For the other less demanding features, i.e. directivity and on-axis field, the errors are generally much smaller. The simplified model provides a similar level of accuracy for all field characteristic (2-6), except the waveform. The fact that both models have similar accuracies can be explained by the fact that the simplified one models just one case, i.e. $\beta_{e}=\beta_{o}$, which is the same as that assumed in the exact model. 
It can also be seen that although the receiver and emitter axes were carefully adjusted, the
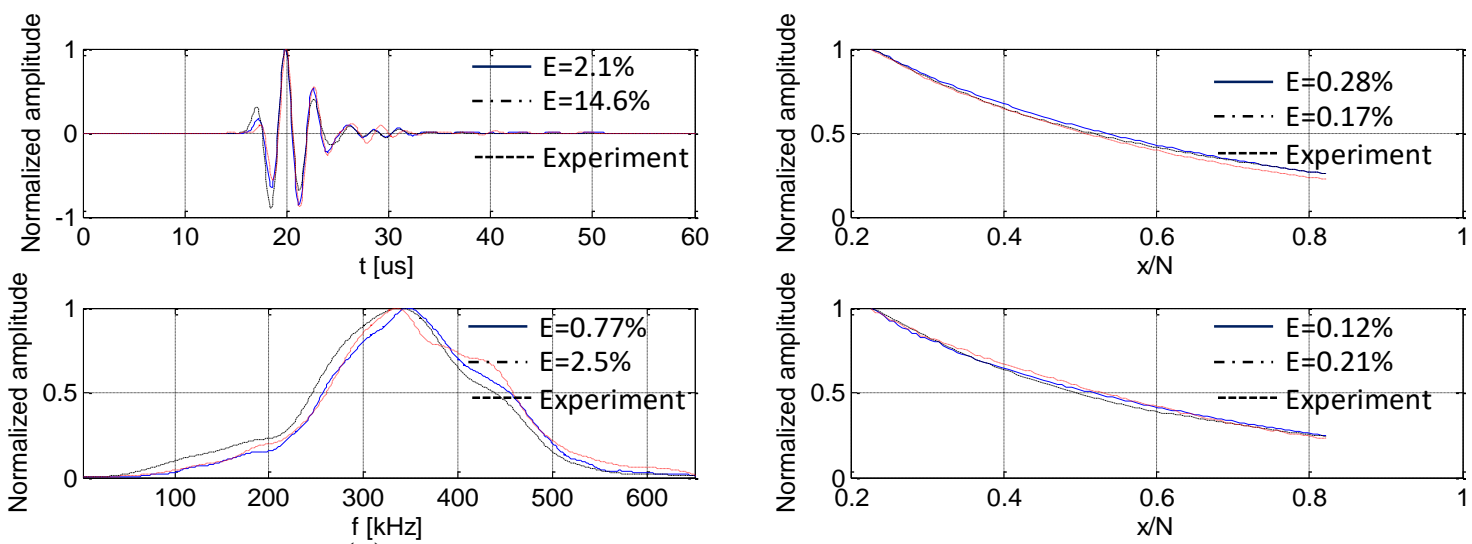

(a)
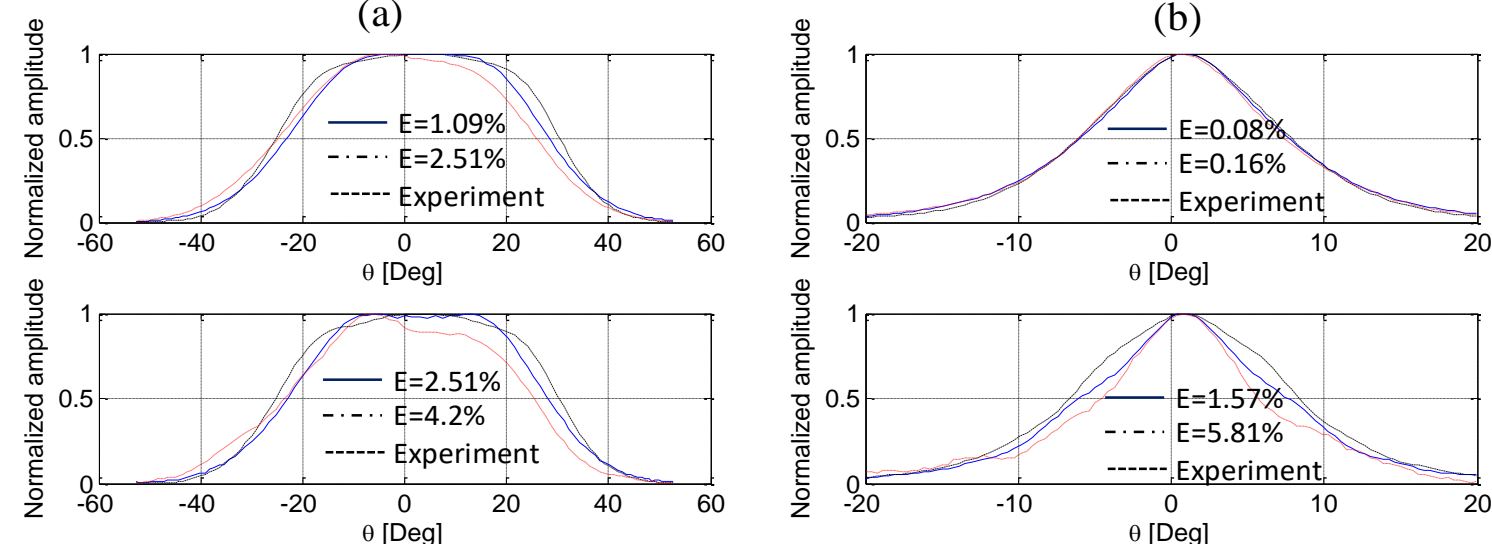

(c)

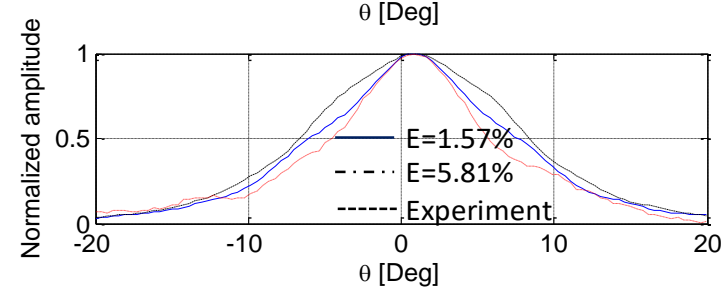

(d)

Fig.7 Comparison between the numerically predicted and experimentally measured field characteristics, together with their associated errors, for the exact and simplified models. The upper plots in each figure show the results obtained from the time-domain signal, whereas the lower plots show the corresponding characteristics determined in the frequency domain. Continue blue line: exact model, dotted/dashed black line: simplified model, dotted red line: experiment. (a) near-field, on-axis impulse response $h_{P E S R}(x / N=0.22, y=0, z=0, t) \quad$ and its spectrum $H_{P E S R}(f) \quad$ (b) on-axis distribution $p_{m}(0.22<x / N<0.81, y=0, z=0)$ and its fixed-frequency counterpart for $f_{o}=350 \mathrm{kHz}$; (c) directivity $p_{m}(x / N=0.22, \theta)$ and its counterpart for $f_{o}=350 \mathrm{kHz}$; (d) directivity $p_{m}(x / N=0.81, \theta)$ and its counterpart for $f_{o}=350 \mathrm{kHz}$.

experimental setup was slightly asymmetric, especially for the case of the directivity pattern measurements in the near field. This phenomenon was also observed in REF (where the same emitter and receiver were used), and can obviously lead to higher prediction errors, especially in the near field.

Three-dimensional representations of the predicted and measured fields are shown in Figs. 8 and 9. Analysis of these results reveals that, similarly to the classical case of a near-field 


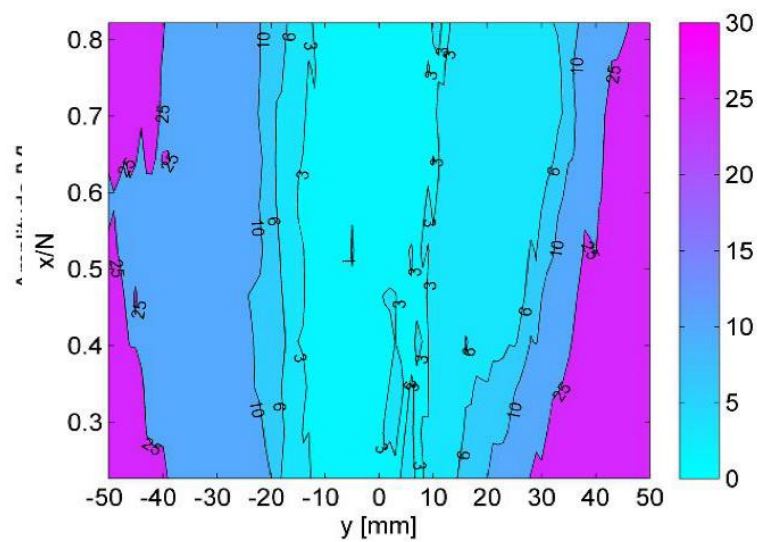

(a)

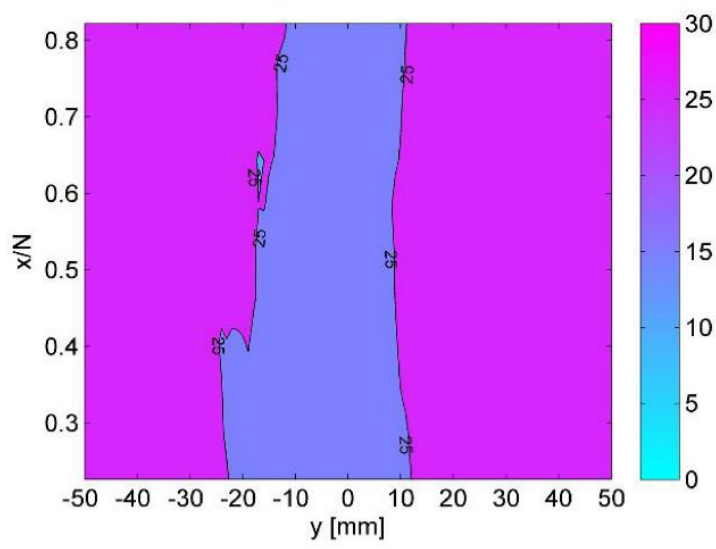

Fi

(c)

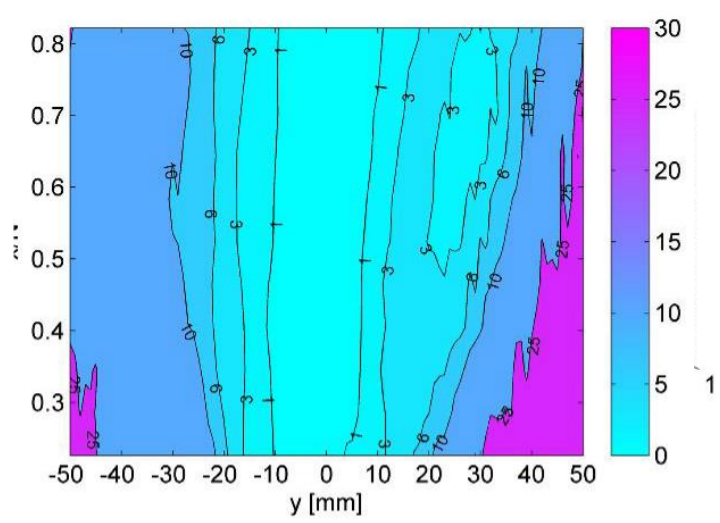

(b)

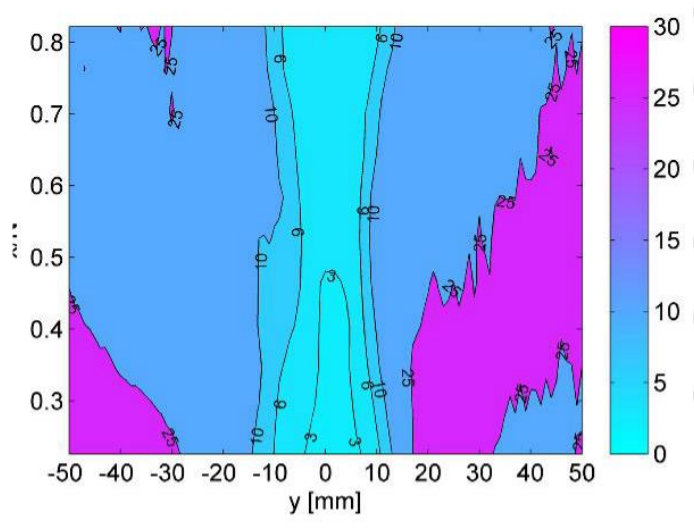

(d)

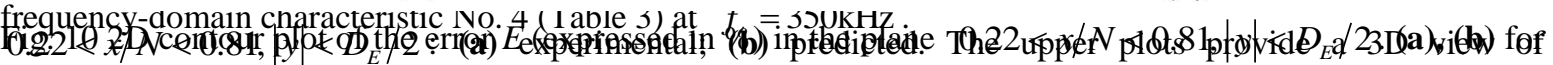

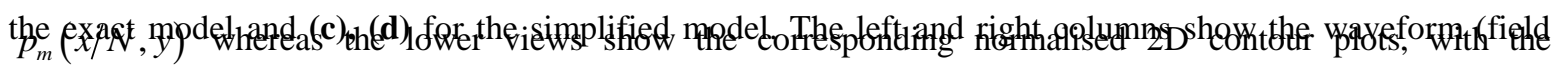
characteristic No. 1) and spectral (field characteristic No. 2) errors, respectively. amplitude expressed in decibels.

region [80], the strongest signal is located in the zone defined by $-L / 2<y<L / 2$, where $L=D_{E}$ is the length of the equivalent linear source (Fig. 5). It is interesting to note that the signal decreases monotonically along the $\mathrm{x}$-axis, which is unusual for an acoustic near field. This is caused by the very strong the attenuation produced by Plexiglas, which is the dominating factor in this setup (see Appendix B). The time- and frequency-domain attributes provide very similar results. The aforementioned asymmetry of the experimentally recorded field can also be noted. 
Finally, Fig. 10 presents the most important parameter for this study, the prediction error of the developed model. The error of the exact model is generally smaller than $3 \%$ in the region close to the $x$-axis, and is even smaller in the frequency domain (as shown in Fig. 7). The asymmetry visible in this figure is caused by the asymmetry of the experimentally recorded field. It is important to note that, within the error margin of 3\%, the predicted waveforms are quite similar to those observed for the experimental signal (Fig.7(a)). This shows that the results obtained with our model are very satisfactory. It also can be seen that the error increases when the receiver moves away from the $x$-axis, where the signal is both weaker and noisier. The signal-to-noise ratio (SNR) of the recorded signal was measured and correlated with the error. The SNR is found as [81]:

$$
S N R_{d B}=20 \log _{10}\left(\frac{p_{r m s}}{n_{r m s}}\right)
$$

where $p_{r m s}$ and $n_{r m s}$ denote the root-mean-square values of the signal and the noise, respectively. (The above formula is a simplified one (because a received signal is always noisy) and is only valid when SNR is relatively high). The value of $p_{r m s}$ was computed within the window shown in Fig. 6(b), and $n_{r m s}$ was determined using the window placed before the arrival time of $h_{p E S R}(\mathbf{M}, t)$ (where only noise is present).

The resulting SNR and corresponding waveform errors are shown in Figs.9 (a) and (b), as a function of the off-axis distance $y$, for two along-axis distances $x / N=0.21$ and 0.81 . Figs. 10 (c) and (d) plot the relationship $E=f(S N R)$. Indeed, although at the greater distance $x / N=0.81)$ the relationship $E=f(S N R)$ is quite linear, in the vicinity of the emitter it is more sensitive to the signal quality and a non-linear relationship is observed. This experiment confirms the direct relationship between the error and the noise level, which allows us to assume that the prediction accuracy is close to $3 \%$ throughout the modeled region. It is interesting to note that since the use of a chirped input signal improved the SNR by approximately $25 \mathrm{~dB}$, in the absence of this technique the experimental error would be driven mainly by the noise induced by the experimental setup, rather than the accuracy of the computational model. The $S N R$, must be greater than 40-50 dB for the influence of background noise to be neglected.

Similarly, as could be expected from the comparisons shown in Fig. 7, the simplified model leads to a significantly stronger error for the waveform (close to $15 \%$ in the near-axis 
region), and a relatively small error (less than 3\%) in the frequency domain. This outcome

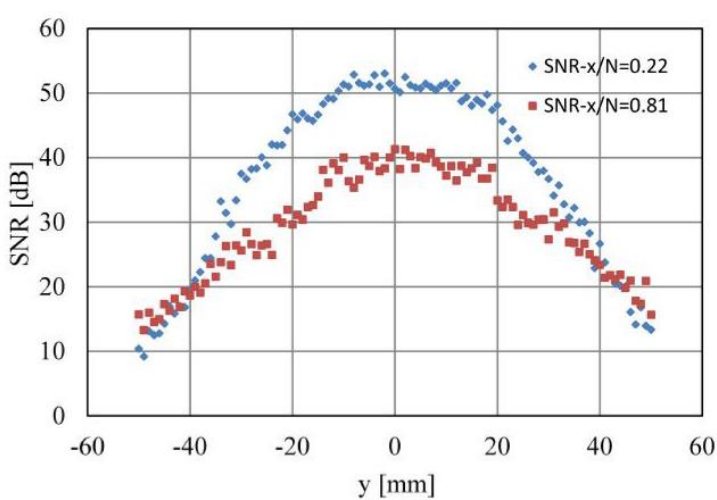

(a)

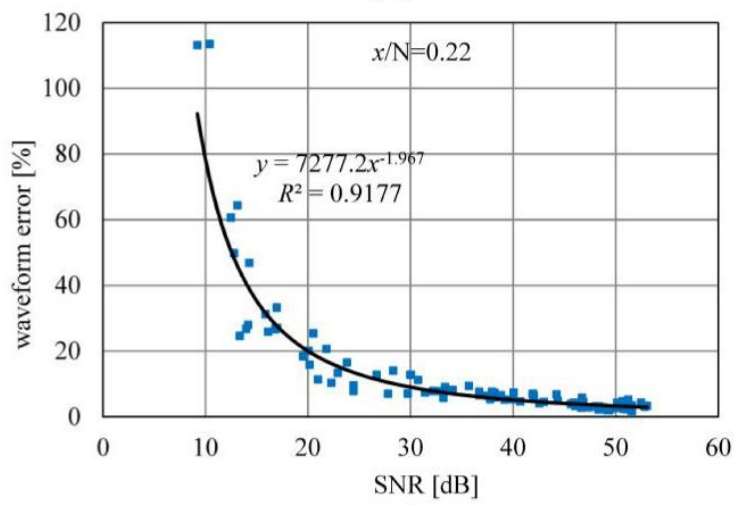

(c)

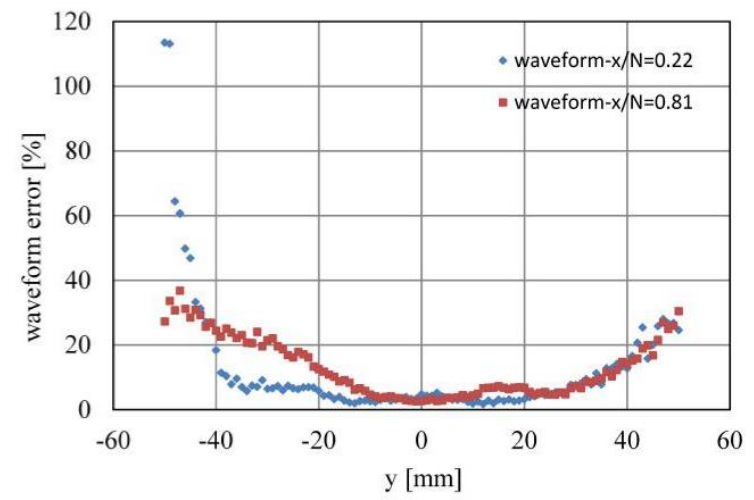

(b)

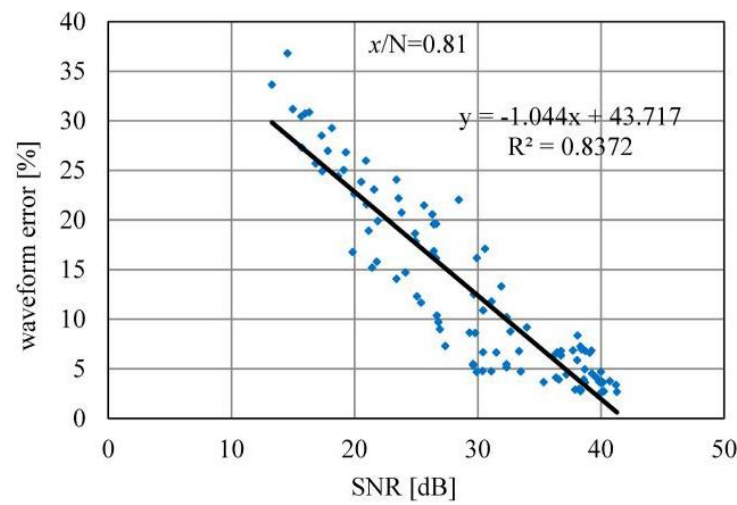

(d)

Fig. 11 SNR (a) and waveform error $E$ (b) for the field characteristic No. 1 as a function of off-axis distance $y$, for two axial distances: $x / N=0.22$ and $x / N=0.81$; waveform errors $E=f(S N R)$ for the axial distances $x / N=0.21$ (c), and $0.81(\mathbf{d})$, respectively, and their corresponding correlation coefficients.

shows that the simplified computation has a more significant impact on the signal's shape than on its amplitude.

\section{Computational recommendations}

\subsection{Influence of the size of surface $S_{1}$ on the computational accuracy}

The SW is excited by a source located inside the ellipse $S_{1}$, which is usually considered to be the projection of emitter $S_{0}$ on the sample surface [23, 24, 82]. Meanwhile, as a consequence of diffraction-induced beam spreading, the dimensions of surface $S_{1}$ do not exactly correspond to the direct projections of the emitter, as could be concluded from Fig. 5. It 
is thus important to determine the influence of the effective area of $S_{1}$ on the computational accuracy.

This influence is analyzed here with the prediction tool, and is quantified using Eq. (35) and by varying $D_{S_{1}}$, the diameter of $S_{1}$ which is assumed to be a circular. The case where $D_{S_{1}}=D_{E}$ is used as a reference. From Fig. 12(a) it can be seen that when $D_{S_{1}}>D_{E}$, the influence of $D_{S_{1}}$ on the field characteristics practically disappears. At the same time, as the amplitude of the received waveform reaches its maximum value, the effective shape of $S_{1}$, which includes most of the signal energy, is very close to a circle of diameter $D_{E}$. For $D_{S_{1}}>D_{E}$ a small improvement in accuracy is obtained (although more computational time is needed), thus demonstrating that the field outside the projection of $S_{0}$ has only a small influence on the results. Indeed, a previous study [83] clearly shows that the field observed very close to the source constitutes its image. Quantitatively, this leads to the requirement that $H_{E} \square N_{f E}[84]$

$$
H_{E} \square N_{f E}=D_{E}^{2} /\left(4 \lambda_{o f}\right)=644 m m
$$

where $N_{f E}$ is the far-field limit of the emitter and $\lambda_{o f}=c_{f} / f_{o} \approx 1 \mathrm{~mm}$ is the wavelength in air at frequency $f_{o}$. The above condition is largely fulfilled in the model (where the surface $S_{1}$ is located in the vicinity of the emitter $\left(H_{E}=15.9 \mathrm{~mm}\right)$.

\section{$\underline{5.2 \text { Influence of } S_{2}}$}

If $S_{2}$ is considered to be the projection of the receiver surface $S_{3}$, then it also tends towards a circle of diameter $D_{R}$. The influence of $S_{2}$ on the computational accuracy as a function of $D_{S_{2}} / D_{R}$, where $D_{S_{2}}$ is the diameter of $S_{2}$ (assumed to be a circle), is plotted in Fig. 12 (b) and (d). This shows that when $D_{S_{2}}>1.5 D_{R}$, the influence of $S_{2}$ becomes negligible and the amplitude of the received waveform reaches its maximum value. When compared with the previous case, now $H_{R}=23.3 \mathrm{~mm}$ and the receiver far field $N_{f R}$ yields:

$$
N_{f R}=D_{R}^{2} /\left(4 \lambda_{o f}\right) \approx 25 m m
$$

It can thus be seen that now $H_{R} \approx N_{f R}$, which means that condition (37) is not fulfilled and the receiver "sees" a surface $1.5 D_{R}$ larger than its geometrical projection. 
Our analysis of field characteristic (not presented here) shows that in cases where

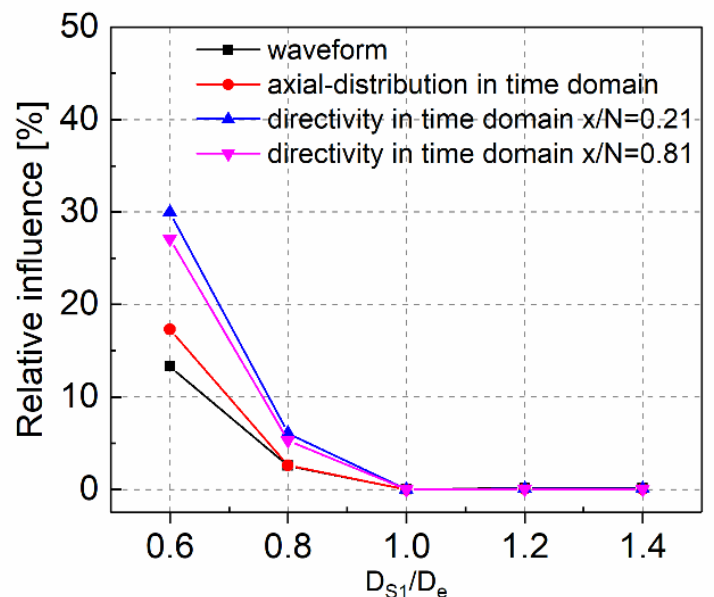

(a)

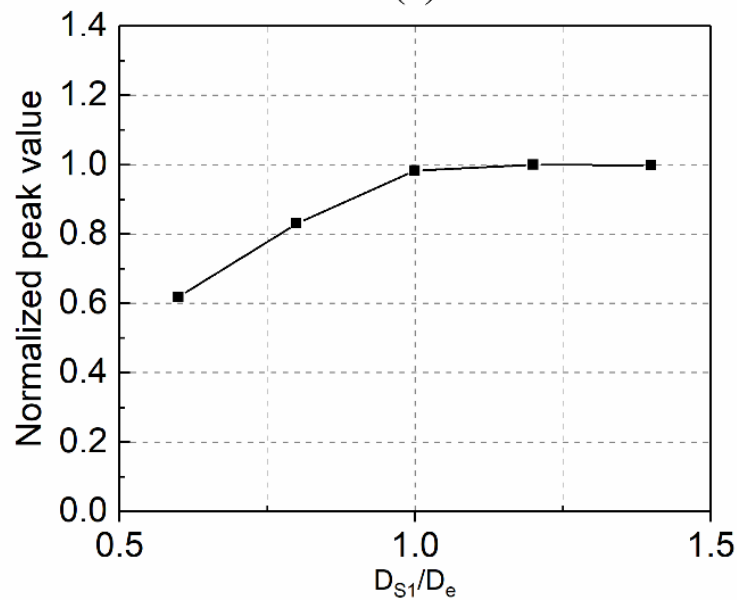

(c)

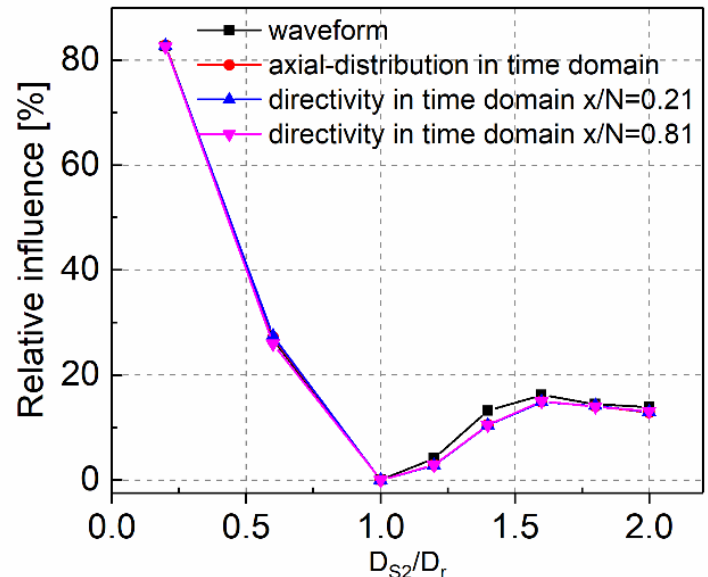

(b)

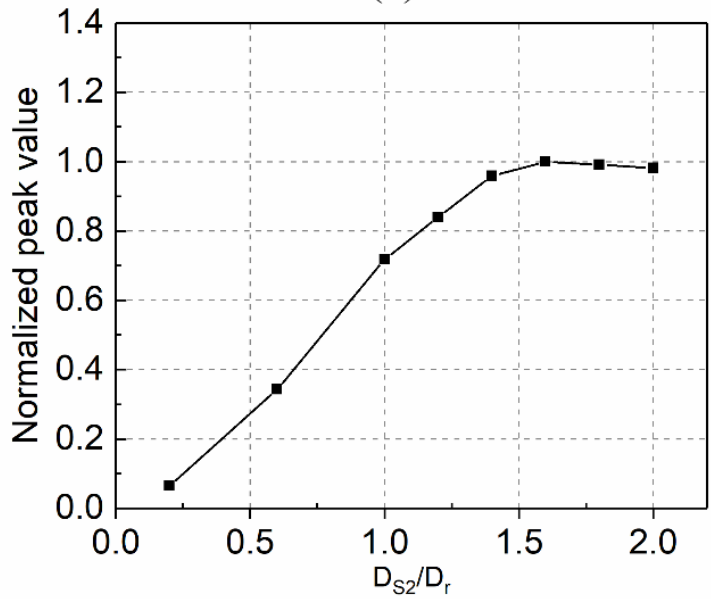

(d)

Fig. 12 Influence of the dimensions of $D_{S_{1}}$ and $D_{S_{2}}$ on the computational accuracy. (a), (b) Influence computed for the same time-domain field caracteristic as that assumed for Fig.7; (c), (d) peak value of the waveform: $p_{m}(x / N=0.21, y=0)$

$D_{S_{2}}<1.5 D_{R}$ the shapes of the waveform and its spectrum do not change, and the only observed influence is that of an increasing signal level (see Fig.12(d)). The condition $D_{S_{2}}>1.5 D_{R}$ thus ensures that the accuracy remains unchanged, and output signal level is maximal.

The preceding discussion shows that the dimensions of $S_{1}$ and $S_{2}$ are generally a function of their distance from the emitter and receiver.

\subsection{Influence of attenuation in air on the computational accuracy}




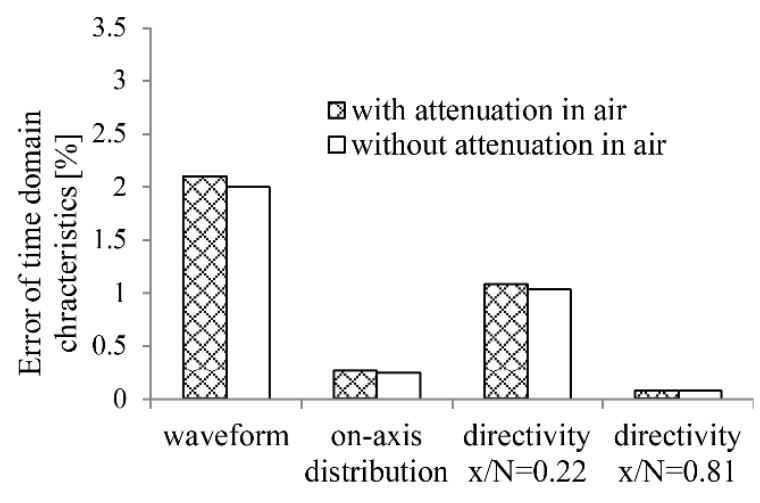

(a)

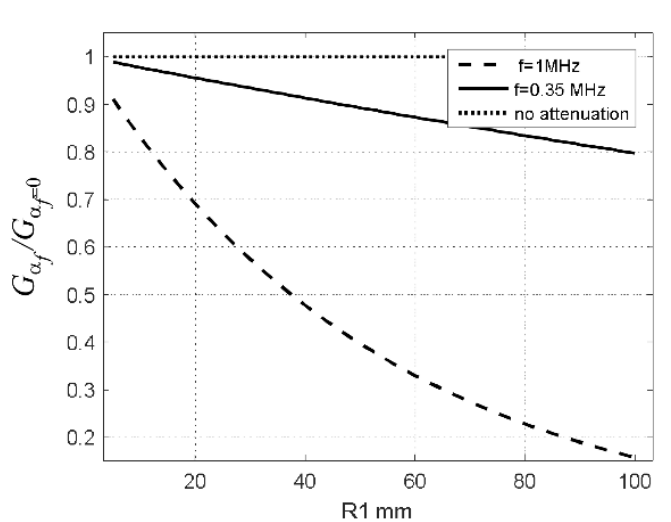

(b)

Fig. 13 Influence of attenuation in air. (a) Field characteristics with and without attenuation in air, with the errors being computed for the same four cases as in Fig. 7; (b) Absolute value of Green's function for a lossy

The influence of attenuation depends not only on $\alpha(\omega)$ (as often commented), but also on the path $R_{1}$ travelled by the signal, and thus on the product $\alpha(\omega) R_{1}$ (Eq. (3)). In order to study this phenomenon, steps 1 and 3 were computed with and without attenuation in air (by assuming $\alpha_{f 0}=0$ in Eq. (3)), and the results are presented in Fig. 13. It can be seen that only very small differences are introduced when the attenuation in air is ignored, thus suggesting that operation (4) could be deleted, with the advantage of reducing by a factor of 30 the computational time involved in steps 1 and 3 (see Table 4). However, this conclusion is valid for the examined case only. Fig. 13(b) plots the influence of attenuation in air by comparing the modulus of the Green's function (Eq. (3)) for lossy and lossless air. This clearly shows that in the context of the present 
medium (Eq. (3)), normalized with respect to its counterpart for air with no losses and plotted as a function of the distance $R_{1}$ from the emitter, at frequencies $f=0.35$ and $1.0 \mathrm{MHz}$, and for $\alpha_{f 0}=0$.

study ( $f=350 \mathrm{kHz}$ ), the influence of attenuation is negligible when $H_{E, R} \approx R_{1}<20 \mathrm{~mm}$. This is confirmed by previous studies reported by Castaings et al. [82], Ke et al. [24], and Delrue et al. [37], who found that under similar conditions the attenuation in air is negligible. However, when the acoustic frequency increases $(f=1 \mathrm{MHz})$ the signal loss caused by attenuation in air is no longer negligible.

\section{Sensitivity of the prediction accuracy to medium parameters}

When the prediction model is run, the parameters describing the medium, i.e. attenuation in the solid $\alpha_{s 1}$, SW velocity $c_{R}$, and the velocity in air $c_{f}$, must be accurately adjusted. The sensitivity of the prediction model's accuracy to the parameters characterizing the medium is analyzed in the following. For this, each of the above parameters is varied, and the results are compared with the reference results obtained with the correct parameters, presented in Table 2 . The sensitivity is then quantified using expression 35 , with $p_{m}$ now denoting the reference result.

From Fig. 14(a) it can be seen that attenuation in the solid, $\alpha_{s 1}$, mainly affects the axial distribution and far-field directivity.

The results plotted in Fig. 14(b) show that $c_{R}$ has the strongest (and also very similar) influence on the four field features analyzed with the model. This outcome is predictable, since $c_{R}$ affects both the velocity dispersion (Eq. (18)) and the inclination angle $\beta_{o}$ (Eq. (24)).

Fig. 14(c) reveals the influence of the ambient temperature $T^{\circ} \mathrm{C}$, which directly affects the velocity in air $c_{f}\left(c_{f} \approx 331.4+0.6 T\right.$ [77] $)$ as well as the attenuation in air $\alpha_{f 0}$, since $\alpha_{f 0}=C_{1} \sqrt{T / T_{0}}$, where $T_{0}=25^{\circ} \mathrm{C}$ and $C_{1}$ is a constant [44]. This result shows that changes in temperature typical of laboratory conditions or an outdoor experiment, i.e. in the range between $0{ }^{\circ} \mathrm{C}$ and $30^{\circ} \mathrm{C}$, can lead to significant errors, especially at lower temperatures. This information is of considerable practical value since the modeled scanner was used mainly under outdoor conditions.

Finally, Fig. 14(d) plots the influence of the acoustic velocity in air $c_{f}$ only. The results are quite similar to those described in Fig. 14(c), thus confirming that in the present study, the 
attenuation in air has a negligible influence, and that the influence of $T$ on the field characteristics is exerted via the changes it produces in $c_{f}$.

\section{Influence of scanner setting on the recorded signal}

In practice, the inclination angles $\beta_{e}$ and $\beta_{r}$, and the emitter and receiver distances $H_{E}$ and $H_{R}$, are adjusted to values representative of the experimental conditions. For this reason, it is also of interest to determine the influence of these parameters on the results produced by the scanner, and to evaluate the accuracy with which they must be adjusted. This influence is

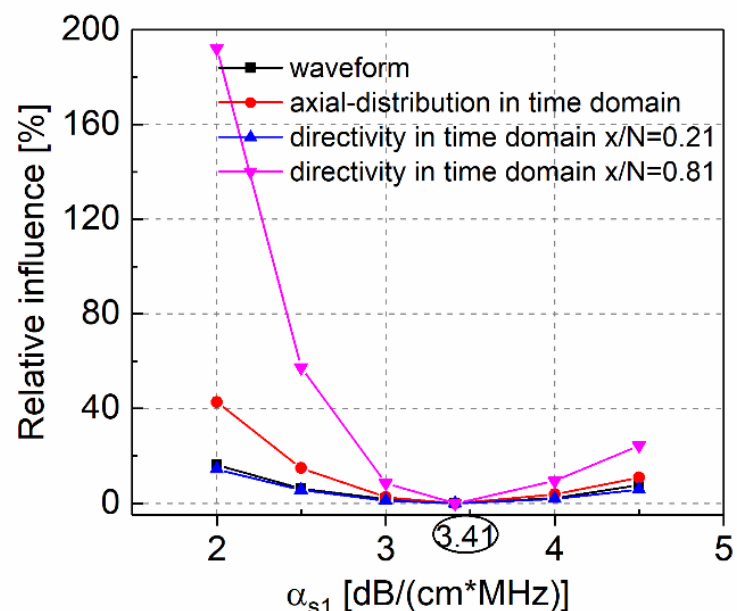

(a)

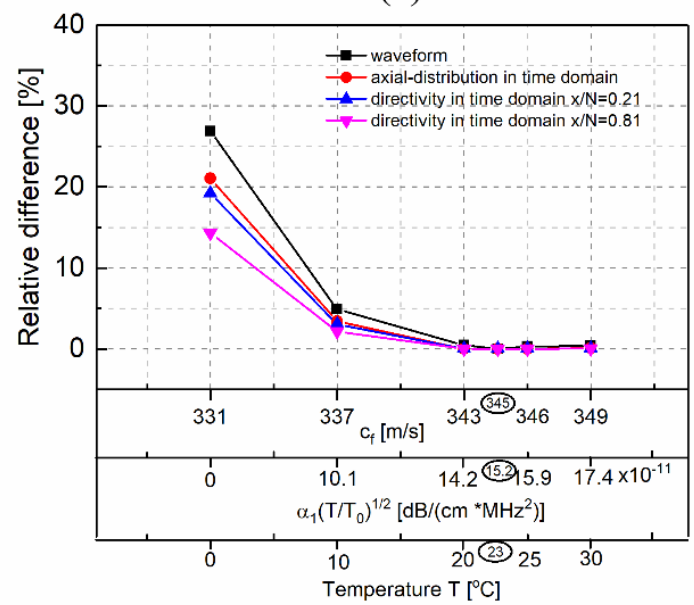

(c)

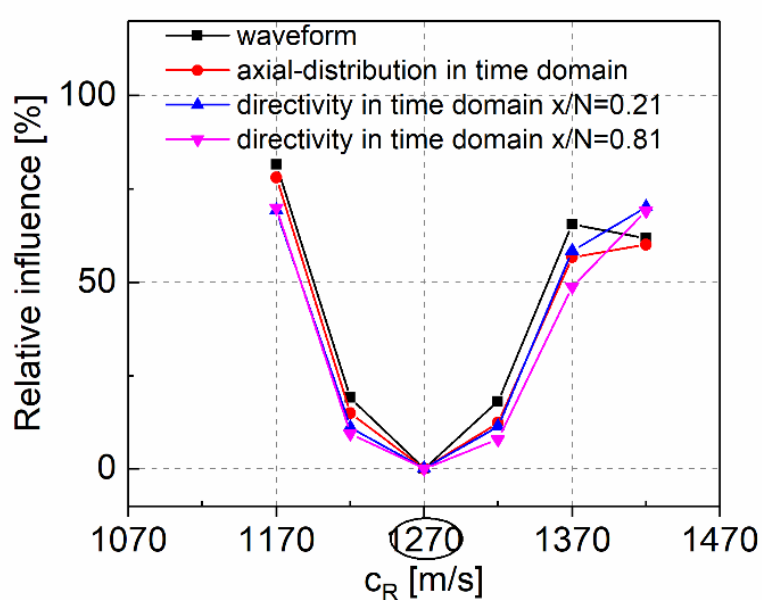

(b)

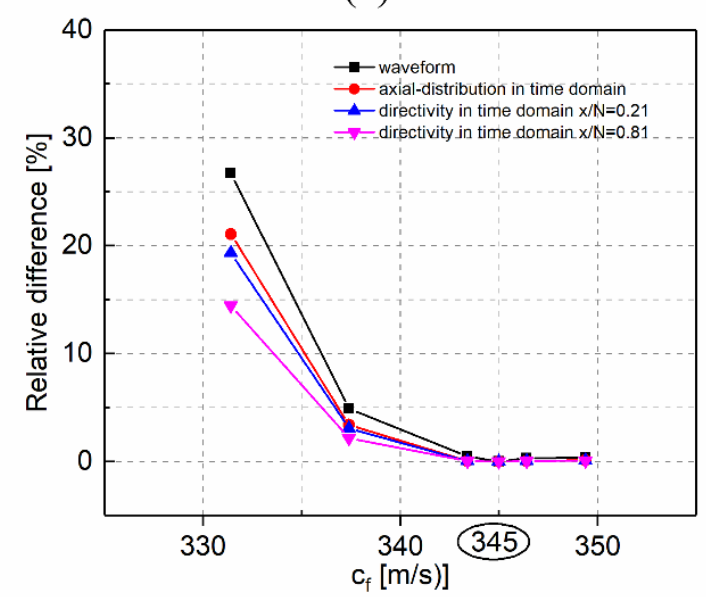

(d)

Fig. 14 Predicted influence of the medium's parameters on the time-domain field caracteristics shown in Fig. 7: (a) influence of attenuation $\alpha_{s 1}$; (b) influence of SW velocity $c_{R}$; (c) Influence of air temperature $T$. (d) Influence of acoustic velocy in air $c_{f}$. The reference values for the relevant parameters (see Tables 1 and 2) are indicated by the encircled numbers on the abscissa of each plot. 
quantified using expression 35, with $p_{m}$ now representing the reference result obtained with the values listed in Tables 1 and 2 .

The results plotted in Fig. 15(a) and (c) show that the emitter angle $\beta_{e}$ has the strongest (and also very similar) influence on the four field features analyzed with the model. This outcome is predictable, since $\beta_{e}$, influences the field shape over surface $S_{1}$ and its effective size (see Appendix B). As the influence of the emitter angle $\beta_{e}$ is found to be considerably more significant than the other parameters, it should be adjusted as accurately as possible [12, $35,67,85])$. The predicted results were also found to be in excellent agreement with the experimentally measured functions (Fig. 15 (a) and (c)).

Although the reciprocity theorem predicts that the receiver angle $\beta_{r}$ has the same influence as $\beta_{e}$, the impact of the former angle is found to be much weaker (Fig. 15(b) and (d)). In the case of the present study, this discrepancy can be attributed to the receiver dimension being five times smaller than that of the emitter $\left(D_{R} / D_{E}=0.2\right)$. The predicted values of the field characteristics were again very well confirmed by the experimental observations.

Fig.16 shows that the emitter-solid distance $H_{E}$, as well as the solid-receiver distance $H_{R}$ , have a relatively small influence on the measured signal. This result confirms the results obtained in Section 5. 


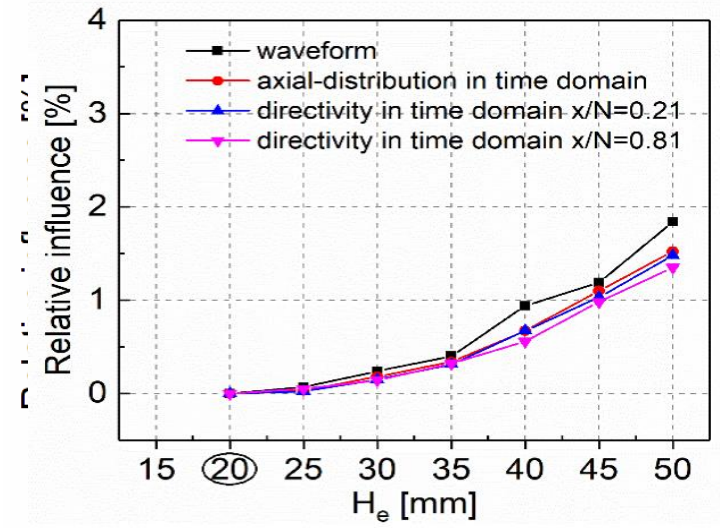

(a)

$\mathrm{Fi}$

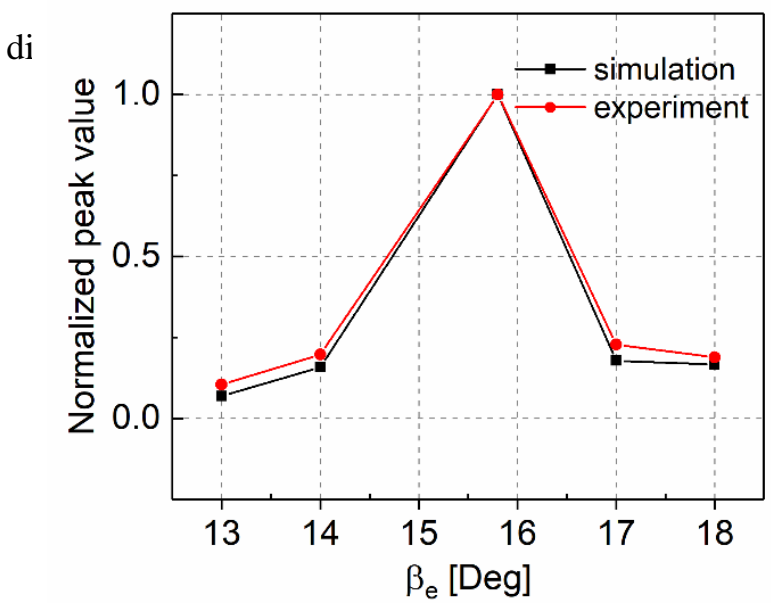

(c)

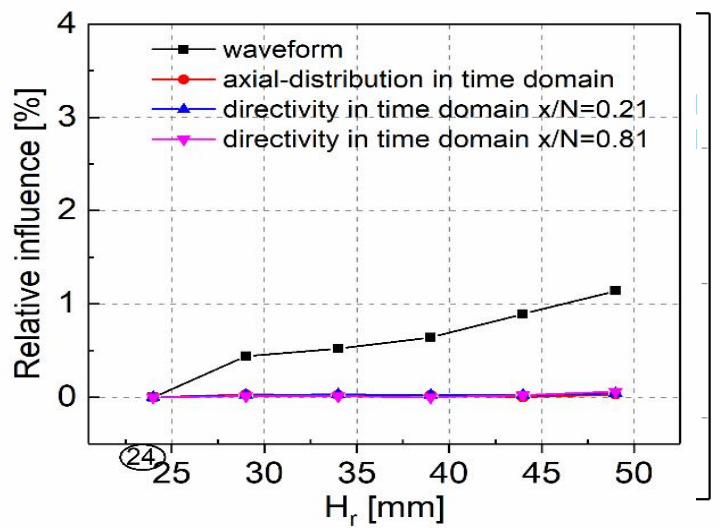

(b)

(b) as

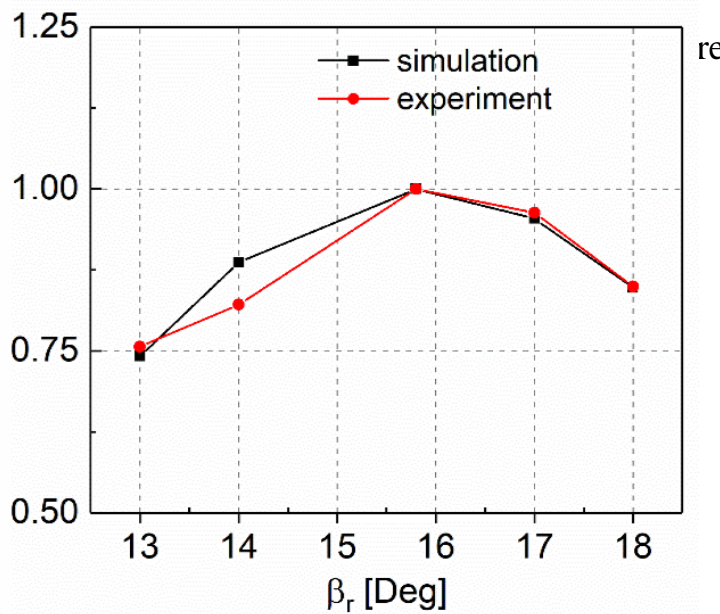

(d)

Fig. 15 (a), (b) Predicted influence of emitter and receiver inclination angles $\beta_{e}$ and $\beta_{r}$ on the four field characeristics shown in Fig. 7, together with the experimental results (for the waveform only). The reference values are indicated by the encircled numbers on the abscissa of these plots. (c), (d) On-axis normalized peak waveform value in the near field $p_{m}(x / N=0.21, y=0)$ as a function of $\beta_{e}$ and $\beta_{r}$. 
indicated by the encircled numbers on the abscissa of these plots.

\section{Computational efficiency}

Various computation times are listed in Table 4 (determined using a computer with an Intel i7 CPU @ 3.7 GHz, 16GB RAM). It can be seen that when the attenuation in air is neglected, the gain in time is not significant, because step 2 is the most time consuming operation (when attenuation in the solid is modeled). When the simplified model is used, step 1 is omitted and step 2 is significantly shortened because the surface integral (15) is replaced by a 1D integration (B.6), which shortens the overall computational time by a factor of ten.

The computing time needed to evaluate a 3D FEM model is approximately 1 month (with the computations being performed for all volume $(150 \times 100 \times 100 \mathrm{~mm})$ including the emitter, receiver and scanned area and with same spatio/temporal resolution. The advantage of the model presented in this study is that a typical parametric analysis (requiring for example 100 field points) can be carried out in just a few hours.

The computation time is also the function of the model size, that is of the number of the elementary surfaces $\Delta x, \Delta y$ used to discretize the surfaces $S_{0}, S_{1}, S_{2}, S_{3}$ and of the vector length $N_{t}$ containing the computed response $h_{p E S R}(t)$. The results reported in Table 4 were obtained for $N_{t}=8192$ (i.e. output response length was set as $\left(N_{t}-1\right) / f_{s}=819.1 \mu \mathrm{s}$ ). The strong influence of length $N_{t}$ on the computational efficiency is illustrated in Table 5 (attention: $N_{t}$ affects also the spectral resolution).

"The computational efficiency could still be improved through the use of the computational parallel algorithm. Taking into account the serial structure of the proposed model (which should be maintained during computing), the parallel computation could be used for consecutive computing of integrals over the surfaces $S_{o} S_{1}, S_{2}$, and $S_{3}$ ”.

Table 4 Computational efficiency (for number of samples of computed response $N t=8192$ )

\begin{tabular}{|l|c|c|c|c|c|}
\hline Version & Step 1 & $\begin{array}{c}\text { Steps 2-3 } \\
1 \text { field } \\
\text { point } \\
(\mathrm{min})\end{array}$ & $\begin{array}{c}\text { Total time } \\
100 \text { field points } \\
(\mathrm{min})\end{array}$ & $\begin{array}{c}\text { Total time } \\
\text { 3D plot as Fig.10 } \\
1100 \text { field points } \\
(\text { day })\end{array}$ & $\begin{array}{c}\text { Total FEM time } \\
\text { Inspected volume with same } \\
\text { Spatio/temporal resolution } \\
1 \mathrm{~mm} / 1 \mu \mathrm{s}\end{array}$ \\
mesh $15 \times 10^{5}$ cells
\end{tabular}




\begin{tabular}{|l|l|l|l|c|}
\hline FEM & & & & \\
\hline
\end{tabular}

Table 5 Computational efficiency for case "with attenuation in air" as a function of response length $N_{t}$

\begin{tabular}{|c|c|c|c|c|}
\hline Response length & Step1 & Steps 2-3 & Total time & Total time \\
\hline 10 / 44 & 2.4 & 0.13 & 15 & $2.7 \mathrm{~h} \approx 0.1$ day \\
\hline 2048 & 6.6 & 0.38 & 45 & $8.2 \mathrm{~h} \approx 0.3$ day \\
\hline 4096 & 12 & 1 & 120 & $23 \mathrm{~h} \approx 1$ day \\
\hline 8192 & 30 & 3.7 & 400 & $80 \mathrm{~h} \approx 3.3$ day \\
\hline
\end{tabular}

\section{Conclusions}

This research demonstrates that a computational approach based on the Rayleigh integral, which predicts the ultrasonic field in a three-step process, allows a complex system such as that of a non-contact SW scanner to be very accurately modeled. The model takes the finite size of the aperture receiver, the attenuation in both air and the tested solid sample, as well as the electric response of the emitter-receiver set $h_{e}$ into account. The accuracy is evaluated quantitatively within the region of practical interest, by comparing the predicted and experimentally measured acoustic fields.

The error obtained for the most demanding field characteristic is generally smaller than $3 \%$ but increases when the point of observation moves away from the system axis. However, it is shown that this increase in error is directly related to a decrease in $S N R$, which must be greater than 40-50 dB for the influence of background noise to be neglected. Overall, it appears safe to assume that an accuracy of $3 \%$ can be achieved throughout the examined area. The prediction errors for less demanding characteristics, such as the on-axis distribution and directivity pattern, are smaller than $0.5-2 \%$. It should be noted that even when the error is close to $3 \%$, the computed and measured signals are virtually identical. The high waveform accuracy obtained is also conditioned by the use of accurate electric response of the emitter-receiver set $h_{e}$, as reported in $R E F$.

Other factors, which can affect the accuracy, include uncertainties in the values of the acoustic velocity and attenuation in the solid sample and air. For this reason, the parameters characterizing the solid were measured experimentally using a specifically developed experimental setup. 
A simplified computational model is also proposed, which neglects the attenuation in air and assumes the emitter inclination angle to be perfectly adjusted, thus allowing the computing time to be shortened by a factor of 10 . The accuracy of this simplified model is comparable with that of the exact model, for all of the studied field characteristics, except the waveform.

Concerning the computational efficiency, the time needed to carry out a typical parametric analysis, involving approximately 100 field points, is close to 7 hours. For the case of the acoustic setup analyzed in this study, it is found that the attenuation in air can be omitted, which allows the computing time of steps 1 and 3 to be reduced leading to an overall reduction of approximately $10 \%$ in the total computing time.

Thanks to this computational performance, the ESR prediction model could be used to investigate the influence of typical settings on the modeled scanner. The system's sensitivity to parameters characterizing the air and the solid was also studied, and the results are expected to be beneficial for the implementation of non-contact scanners for the identification of solid parameters. The analysis of the influence of temperature provides indications for the outdoor use of non-contact devices. The influence of typical user-defined settings, such as the inclination angle and transducer-solid distance, were modeled and have led to recommendations for future use of the scanner.

The model developed in the present study was implemented using the Discrete Representation computational technique, which is very well adapted to the accurate determination of the integrals inherent to this type of solution The computations were carried out for a circular planar emitter and receiver, but can also be straightforwardly adapted to the case of different planar and quasi-planar aperture shapes. In particular, it would not be difficult to model a focused emitter or receiver, by replacing the surface of our planar transducers with spherical or cylindrical surfaces, and to model the emitter or receiver as a multi-element array.

The model is not limited to the special case of Plexiglas, and could also be used for a medium obeying any law of the type $\alpha(\omega)$, provided that the causal solution for $g_{\alpha}$ is known. Apart from the fact that the model is not limited to air-coupled systems, replacement of the edge material by a solid could also be used to model semi-contact SW scanners equipped with contact wedge transducers. This method can be also adapted to the modeling of a system relying on Lamb wave propagation, provided the corresponding dispersion characteristic is known. 
Moreover, if the displacement field on the sample surface $S_{2}$ is considered to be the output, it would be straightforward to model air-coupled systems equipped with an interferometric receiver. Similarly, it would also be straightforward to model a pulsed laser source, or the effects of mechanical impact source, by allowing the point-like force at surface $S_{1}$ to represent the input excitation.

\section{Acknowledgements}

The China Scholarship Council (CSC), the French National Agency for Research (ANR, projects "SENSO" and "ENDE) "and Ecole Centrale de Lille are kindly acknowledged for their partial financial support of this research program. The authors also wish to thank the reviewers for their constructive comments. 


\section{Appendix A Measurement of medium parameters}

In the modeling of an ESR system, the basic properties of a tested solid medium, i.e. SW velocity $c_{R}$, attenuation in the solid $\alpha_{s}$, and velocity in air $c_{f}$ should be accurately known. Although these parameters can be found in the literature, they were determined experimentally in the present study, in order to optimize the accuracy of the prediction model.

Attenuation measurements should normally be performed in the far field of the emitter, in order to avoid errors encountered in the near field, which require so-called diffraction corrections $[86,87]$. For this reason, the same experimental system was used as that described in Section 4, with the exception of surface $S_{1}$, which was reduced in size, such that $S_{1}=\Delta S_{1}$ (Fig. A.1). The reduced source was created by placing a plastic mask, with a square hole of dimension $D_{S_{1}}=10 \mathrm{~mm}$, on the surface of the Plexiglas. From Eq. (26) the near-field limit then becomes:

$$
N^{\prime}=\left(D_{S_{1}}\right)^{2} /\left(4 \lambda_{o R}\right)=6 \mathrm{~mm}
$$

and the attenuation measurements can be performed without diffraction corrections, at distances $x>N^{\prime}$.

Combining the conclusions developed in the core of this paper and Appendix B, we can assume that the attenuation in air is negligible, and since $S_{2}$ is close to $S_{3}$ propagation from $S_{2}$ to $S_{3}$ can be reduced to a simple delay $t_{3}=R_{3} / c_{f}$. By assuming that $S_{1}=\mathrm{d} S_{1}=\Delta S_{1}$ in Eq. (20), and combining Steps 2 and 3, the response given by Eq. (23) can be approximated to:

$$
h_{p E S R}(\mathbf{M}, t)=\frac{\mathrm{d}^{2}}{\mathrm{~d} t^{2}} C F_{n e}\left(\mathbf{M}_{1}, t\right) * g_{\alpha_{s}}\left(\mathbf{M}_{1}, \mathbf{M}_{2}, \alpha_{s}, t\right) \Delta S_{1} * \delta\left(t-t_{3}\right)
$$

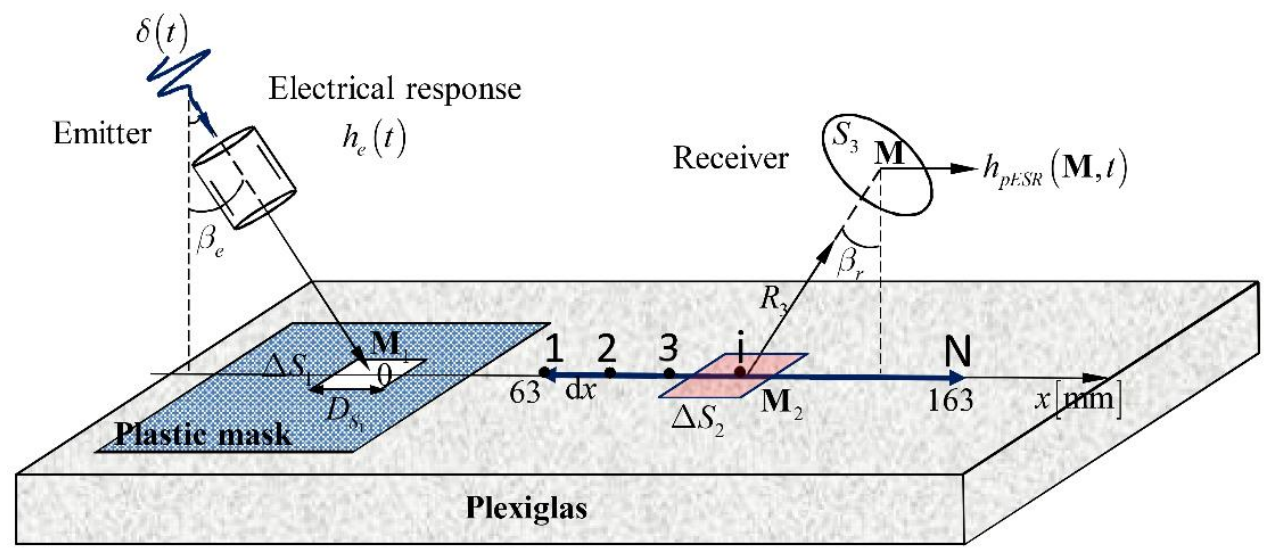

Fig. A.1 Illustration of the experimental set-up and notations used to determine $\alpha_{s}(\omega)$ and $c_{R}(\omega)$ 
where $C$ is a constant. By expressing Eq. (A.1) in the frequency domain and assuming that $R_{2}=\left|\mathbf{M}_{2}-\mathbf{M}_{1}\right|=x$, Eq. (18) can be combined with Eq. A.1 to yield:

$$
H_{p E S R}[\mathbf{M}(x), j \omega]=\mathcal{F}\left\{h_{p E S R}[\mathbf{M}(x), t]\right\}=-\omega^{2} C F_{n e}[\mathbf{M}(x), j \omega] \frac{e^{-\alpha_{s}(\omega) x-j k_{s} x}}{2 \pi \sqrt{x}} e^{-j k_{f} R_{3}}
$$

where $F_{n e}(j \omega)$ is the complex spectrum of normal forces exciting $\Delta S_{1}, k_{s}=\omega / c_{R}(\omega)$ and $k_{f}=\omega / c_{f}$ are the wavenumbers in Plexiglas and air, respectively, and $R_{3}=\left|\mathbf{M}-\mathbf{M}_{2}\right|$. The above expression shows that the leaky wave propagation and reception is now reduced to a travel path phase shift $k_{s} x+k_{f} R_{3}$, and that the spectral amplitude of the received signal thus depends on the travelled distance $x$ only, as well as the attenuation in Plexiglas $\alpha_{s}$. Finally, the spectral amplitude of the temporal SW pulse $h_{p E S R}[\mathbf{M}(x), t]$ recorded as a function of position $x$ can be expressed as:

$$
A\left(x, \alpha_{s}, \omega\right)=\left|H_{p E S R}[\mathbf{M}(x), \omega]\right|=C_{1} \omega^{2} F(\omega) \frac{1}{\sqrt{x}} e^{-\alpha_{s}(\omega) x}
$$

where $C_{1}$ is another constant. The above expression is often used to express SW attenuation [13].

Measurements of $h_{p E S R}[\mathbf{M}(x), t]$ were carried out using the same excitation as that described in Section 4, along a line defined by $63<x<163 \mathrm{~mm}$ (far field), for $n=100$ points and a step $\mathrm{d} x=1 \mathrm{~mm}$ (in order to respect the spatial sampling condition: $\mathrm{d} x / \lambda_{o R}<2$ ). The resulting $100 \mathrm{SW}$ signals are shown in Fig. A.2 (a), and the corresponding spectra $A\left(x, \alpha_{s}, \omega\right)$ in Fig. A.2(b). For the $350 \mathrm{kHz}$ emitter (identical to that used in the core of this paper), the resulting central frequency is, as expected, close to $f_{o}=350 \mathrm{kHz}$ and the corresponding useful frequency band $B$ is estimated to be $200-500 \mathrm{kHz}$.

This experiment also allowed us to simultaneously adjust the receiver inclination angle $\beta_{r}$, since the correct angle provides the maximum signal level (see Fig.15.d) (the emission angle $\beta_{e}$ is irrelevant here). This angle was found to be: $\beta_{r} \approx 15.8^{\circ}$ which was used during all of the experiments and predictions in the present study (Table 1). 


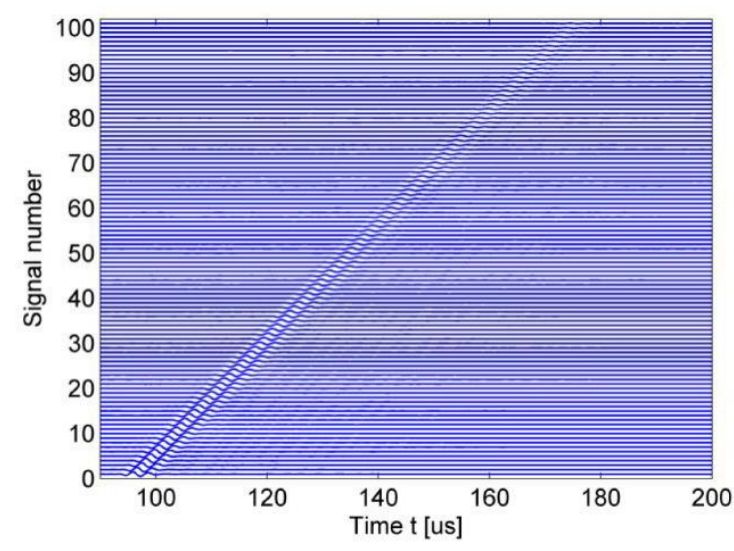

(a)

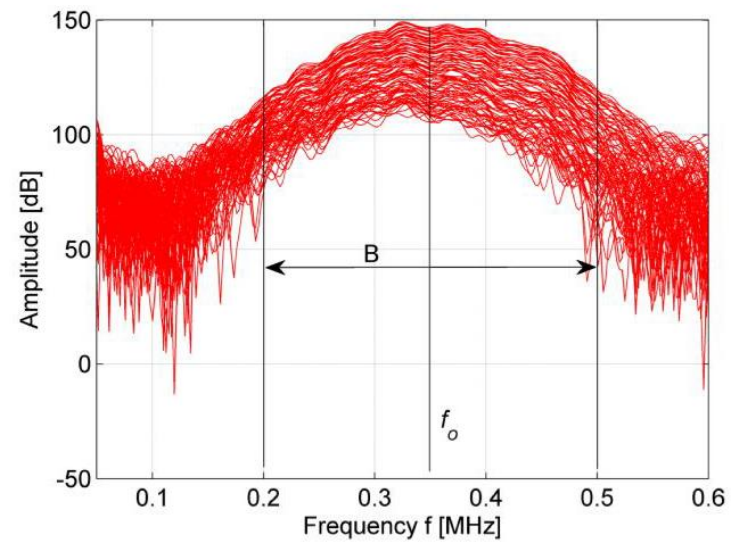

(b)

Fig. A.2 (a) $h_{p E S R}[\mathbf{M}(x), t]$ pulses measured along the profile $63<x<163 \mathrm{~mm}$ at intervals $\mathrm{d} x=1 \mathrm{~mm}$; (b) corresponding spectra.

\section{A.1 Attenuation}

From Eq. (A.3), the frequency-dependent attenuation of Plexiglas can be found for each pair of signals recorded at distance $x_{i-1}$ and $x_{i}[88]$ :

$$
\alpha_{s}(\omega)_{[\mathrm{Np}]}=\frac{1}{x_{i-1}-x_{i}}\left[\frac{1}{2} \ln \frac{x_{i-1}}{x_{i}}-\ln \left|\frac{A_{i-1}(\omega)}{A_{i}(\omega)}\right|\right]
$$

from which the attenuation expressed in decibels is derived using the relationship $\alpha_{s}(\omega)_{[\mathrm{dB}]}=8.68 \alpha_{s}(\omega)_{[\mathrm{Np}]}$. The attenuation $\alpha_{s}(\omega)$ was computed for all distance pairs $x_{i}, x_{j}$, such that $x_{i}-x_{j}>10 \mathrm{~d} x$, and all of the resulting values were averaged. The resulting relationship between attenuation and frequency is presented in Fig. A.3(a). As could be expected, this reveals the attenuation's quasi-linear frequency dependence within the frequency band $B$, in which the SNR is sufficient to provide robust values. The linear regression adjusted to the linear portion of this graph leads to an attenuation estimated as:

$$
\alpha_{s}(f)_{[\mathrm{dB} / \mathrm{cm}]}=\alpha_{s 1} f+\alpha_{s 0}=3.41 f+0.13
$$

where $f$ is the frequency in MHz. The attenuation at the central frequency $f_{o}$ is thus found to be $\alpha_{s}\left(f_{o}=0.35 \mathrm{MHz}\right)=1.32 \mathrm{~dB} / \mathrm{cm}$.

An alternative method can be used to estimate the attenuation by determining the best fit to the experimental spectral amplitude at the central frequency $A\left(x, \alpha_{s}, f=f_{o}\right)$, using the function $e^{-\alpha_{s}(\omega) x} / \sqrt{x}$ included in Eq. (A.3). The curve with the best fit (shown in Fig. A.3(b)) 


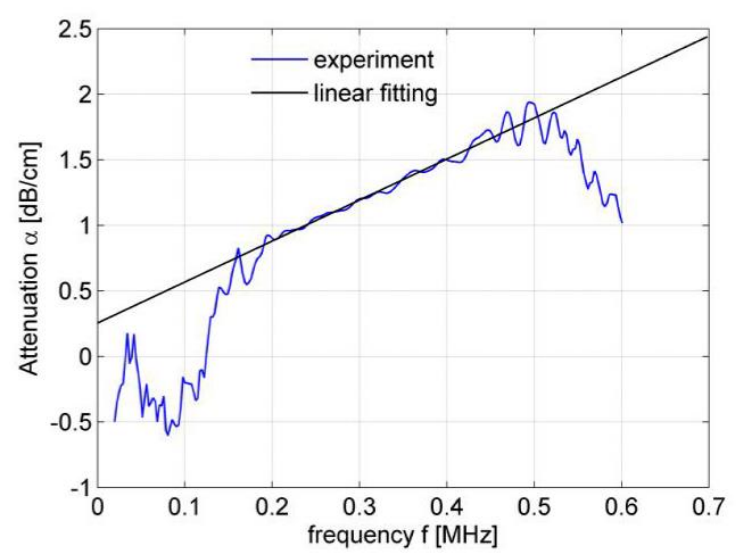

(a)

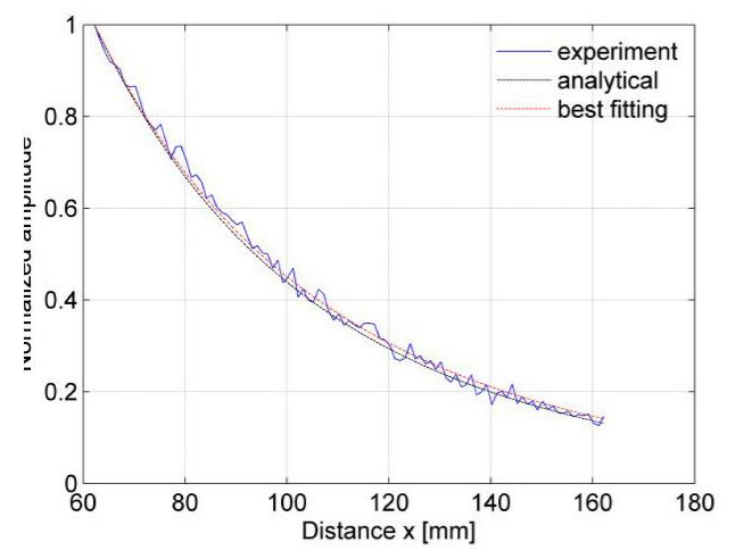

(b)

Fig. A.3 (a) Measured attenuation $\alpha_{s}(\omega)$ and its linear regression of the measured attenuation $\alpha_{s}(\omega)$ over the range $200-450 \mathrm{kHz}$; (b) Spectral amplitude $A\left(x, \alpha_{s}, f=350 \mathrm{kHz}\right)$ measured experimentally, and computed using Eq. (A.5), with $\alpha_{s}=1.32 \mathrm{~dB} / \mathrm{cm}$. The best fit is found for an attenuation equal to $\alpha_{s}\left(f_{o}=0.35 \mathrm{MHz}\right)=1.30 \mathrm{~dB} / \mathrm{cm}$.

leads to: $\alpha_{s}\left(f_{o}=0.35 \mathrm{MHz}\right)=1.30 \mathrm{~dB} / \mathrm{cm}$, which is very close to the aforementioned result of $1.32 \mathrm{~dB} / \mathrm{cm}$ (Fig. 3(a)). These two methods were also used to determine the attenuation in [13].

In a further example, Press and Healy [89] determined the SW attenuation in Plexiglas experimentally, reporting a value of: $\alpha_{s}\left(f_{o}=0.35 \mathrm{MHz}\right)=1.60 \mathrm{~dB} / \mathrm{cm}$, which is somewhat different to our result (perhaps because these authors used a different type of Plexiglas). For the predictions described in Section 4, our experimentally determined expression for the attenuation, given in Eq. (A.6), was used (Table 2).

\section{A.2 Velocity and dispersion of SW in Plexiglas}

The sequence of 100 recorded pulse signals $h_{p E S R}(x, t)$ (Fig. A.2(a)) was processed using a Slant Stack (SL) transformation $[90,91]$ in order to compute the SW dispersion $c_{R}(\omega)$, leading to the results shown in Fig. A.4. It can be seen that the velocity dispersion is very low. Within the studied bandwidth $B(200-500 \mathrm{kHz})$, the velocity varies between $1265 \mathrm{~m} / \mathrm{s}$ and $1275 \mathrm{~m} / \mathrm{s}$. At the central frequency we obtain $c_{R}\left(f_{o}=350 \mathrm{kHz}\right) \approx 1270 \mathrm{~m} / \mathrm{s}$. Theoretically, the attenuation-related dispersion should follow the relationship given in Eq. (18): 


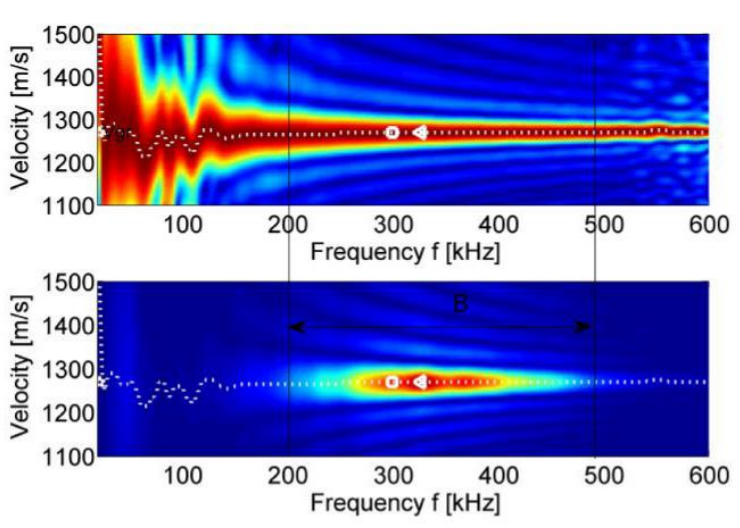

(a)

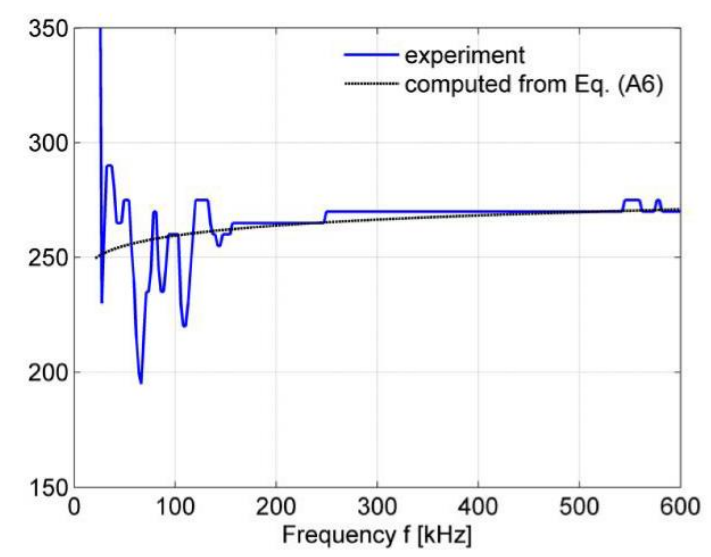

(b)

Fig.A.4. (a) Slant Stack data obtained from the signals shown in Fig.A.3. Upper figure: with corrected amplitude; lower figure: without amplitude correction, showing the usable frequency bandwidth B ; (b) $c_{R}(f)$ obtained experimentally, and computed from Eq.(A.7)) for $c_{R}\left(f_{o}=350 \mathrm{kHz}\right) \approx 1270 \mathrm{~m} / \mathrm{s}$ and $c_{R}\left(\omega_{\infty}\right)=1300 \mathrm{~m} / \mathrm{s}$

$$
c_{R}(\omega)=\frac{1}{-\frac{2 \alpha_{s 1}}{\pi} \ln \left|\frac{\omega}{\omega_{\infty}}\right|+\frac{1}{c_{R}\left(\omega_{\infty}\right)}}
$$

In order to use this expression in the model, we need to determine the parameters $\omega_{\infty}$ and $c_{R}\left(\omega_{\infty}\right)$. Taking $\alpha_{s 1}=3.41 \mathrm{~dB} /(\mathrm{cm} \cdot \mathrm{MHz})$ and assuming the maximum frequency of interest to be $f_{\max }=0.5 \mathrm{MHz}$, and using $\omega_{\infty}=100 \omega_{\max }$ (in order to fulfill the condition $\left.\omega_{\infty} \square \omega_{\max }\right)$, the best fit to the experimental curve leads to: $c_{R}\left(\omega_{\infty}\right)=1300 \mathrm{~m} / \mathrm{s}$. The theoretical value of $c_{R}(\omega)$ computed from Eq. (A.6) is plotted in Fig. A.4 (b), and is indeed very closely matched to the experimental values above $200 \mathrm{kHz}$.

The resulting velocity and dispersion are in very good agreement with the experiments performed by Mattei and Adler [66], who show that the SW velocity in Plexiglas lies in the range between 1260 and $1280 \mathrm{~m} / \mathrm{s}$. The obtained values are retained for the prediction and are summarized in Table 2.

\section{A.3 Velocity in air}

The velocity in air was also determined by applying a SL transform to the signals, using a setup similar to that shown in Fig. A.1, but with the direct emitter-receiver signals being recorded and analyzed (as in $R E F$ ). The velocity obtained was $c_{f}=344 \mathrm{~m} / \mathrm{s}$, thus very close 
to the value of $c_{f}=345 \mathrm{~m} / \mathrm{s}$ found using the formula: $c_{f} \approx 331.4+0.6 T$ [77] at a temperature $T=25^{\circ} \mathrm{C}$. The former value, i.e. $c_{f}=345 \mathrm{~m} / \mathrm{s}$ was retained for the predictions (Table 2)

\section{Appendix B. Simplified model of the ESR system}

The geometry of the air-coupled wedge emitter modeled in Section 1 is shown in Fig. B.1. In the case of wedge transducers, a commonly used approximation is that any pulse emitted from point $\mathbf{M}_{0}$ on $S_{0}$ reaches the corresponding projection point $\mathbf{M}_{1}$ on $S_{1}$ and arrives at the

point $\mathbf{M}_{L}$ located on reference line $L$ along each travel path $\overline{\mathbf{M}_{0} \mathbf{M}_{1} \mathbf{M}_{L}}$. If the inclination angle is set correctly, in accordance with Eq. (24), i.e. $\beta_{e}=\beta_{o}$, it can easily be shown that the signal travel time $t_{0}$ along each travel path $\overline{\mathbf{M}_{0} \mathbf{M}_{1} \mathbf{M}_{L}}$ is constant and equal to:

$$
t_{0}=\frac{\overline{\mathbf{M}_{0} \mathbf{M}_{1}}}{c_{f}}+\frac{\overline{\mathbf{M}_{1} \mathbf{M}_{L}}}{c_{R}}
$$

The above assumption is justified (as already commented in section 5.1 ) if $S_{1}$ is located close to $S_{0}$ (see condition (37)). The field between $S_{0}$ and $S_{1}$ can then be assumed to be a plane-wave collimated beam [84].

Thus, neglecting the attenuation in air, propagation along any path $\overrightarrow{\mathbf{M}_{0} \mathbf{M}_{1} \mathbf{M}_{L}}$ is reduced to a time delay only, and Eq. (1) can be simplified to:

$$
h_{\Psi}\left(\mathbf{M}_{0}, \mathbf{M}_{L}, t\right)=C^{\prime} v_{n}\left(\mathbf{M}_{0}\right) \Delta S_{0}\left(\mathbf{M}_{0}\right) \delta\left(t-t_{0}\right)
$$


where $h_{\Psi}\left(\mathbf{M}_{L}, t\right)$ is the contribution of an emitter element $\Delta S_{0}\left(\mathbf{M}_{0}\right)$ excited by $v_{n}\left(\mathbf{M}_{0}\right) \delta(t)$ and observed at $\mathbf{M}_{L}$, and $C^{\prime}$ is a constant. By introducing Eqs. (9)-(12) the force exciting the SW observed at $\mathbf{M}_{L}$ and originating from $\Delta S_{0}\left(\mathbf{M}_{0}\right)$ can then be written as:

$$
F_{n}\left(\mathbf{M}_{o}, \mathbf{M}_{L}, t\right)=\frac{\mathrm{d}^{2}}{\mathrm{~d} t^{2}} C^{\prime \prime} v_{n}\left(\mathbf{M}_{0}\right) \Delta S_{0} \delta\left(t-t_{0}\right)
$$

where $C^{\prime \prime}$ is another constant. Notice that each point $\mathbf{M}_{L}\left(y_{o}\right)$ collects all of the wavelet contributions emanating from the corresponding line $C D\left(y_{o}\right)$, which in turn originates from an equivalent chord $C_{0} D_{0}\left(y_{o}\right)$ on the emitter's surface. With increasing distance along the $x$ coordinate, all of the contributions are perfectly superimposed (if Eq. (B.1) holds) and the final signal observed at $\mathbf{M}_{L}$ is proportional to the length of the equivalent chord $C_{0} D_{0}(y)$, which can easily be derived as:

$$
W(y)=2 \sqrt{a^{2}-y^{2}},|y| \leq a
$$

where $a=D_{E} / 2$ is the emitter radius. This phenomenon is illustrated in Fig. (B.2), which represents field pulses at three different positions: $x_{1}, x_{2}$ and $x_{L}$, clearly showing that the signal strength increases as a function of $x$ (i.e. proportionally to contributing emitter's surface) Note that the resulting field observed along the line $\boldsymbol{L}$ (Fig. B.1) represents the sum of all of the

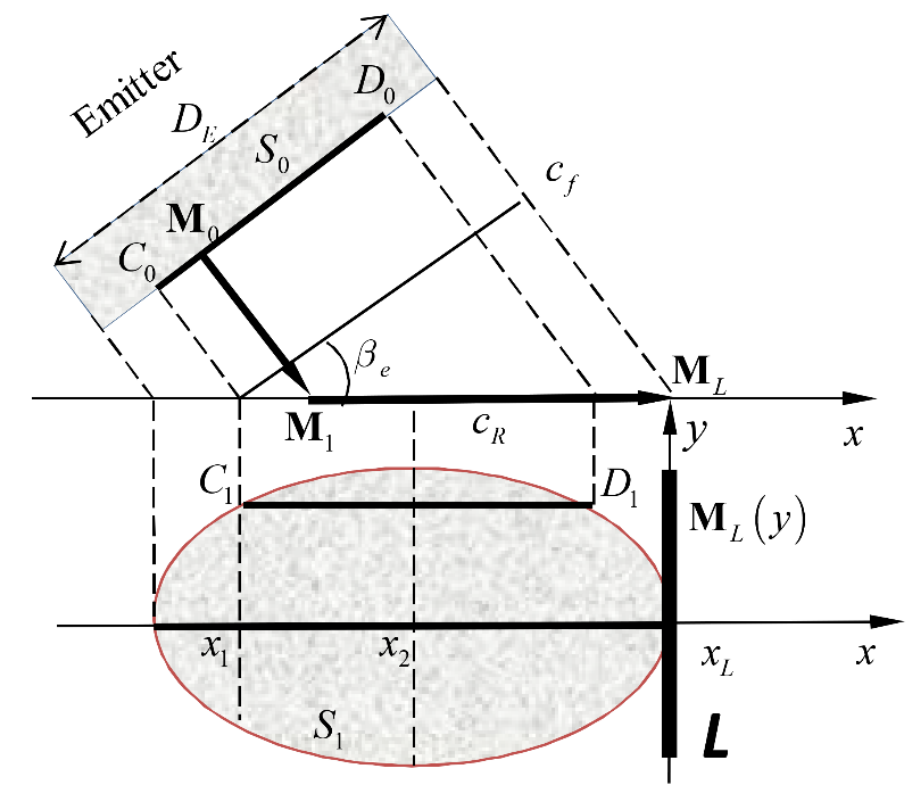

Fig. B.1 Geometry of the modeled air-coupled wedge emitter (see also Fig. 2) 

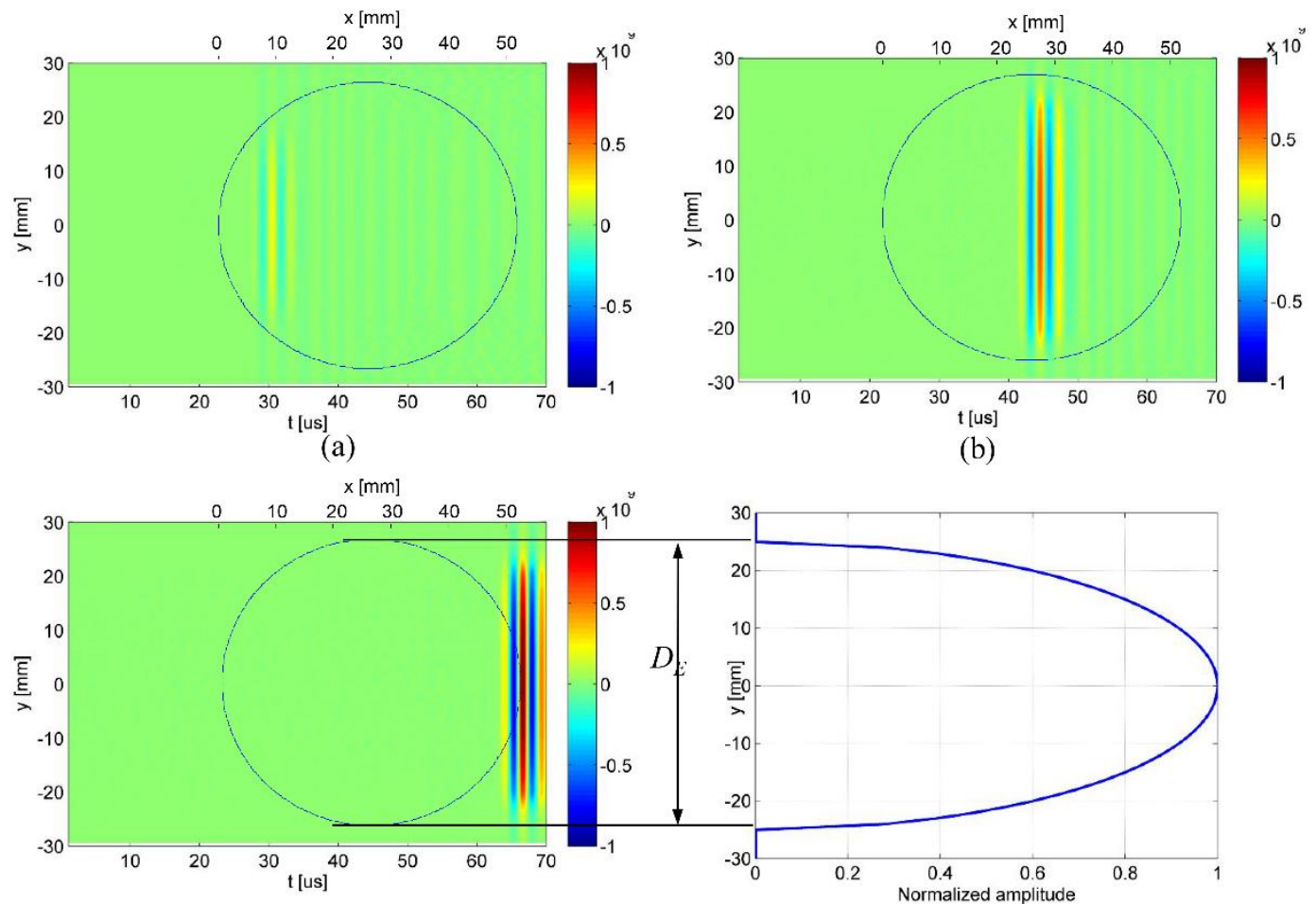

(c)

(d)

Fig.B.2. (a), (b), (c) Snapshots of the field $F_{n}(x, y, t)$ observed at surface $S_{1}$ (indicated by circles), along the $y$ axis, at three positions $x=x_{1}, x_{2}$ and $x_{\mathrm{L}}$ (see Fig.B.1); (d) Normalized weighting function $W(y)$ defined in Eq. (B.4).

superimposed emitter contributions. The force observed at any point $\mathbf{M}_{L}(y)$ on line $\boldsymbol{L}$ is thus proportional to the length of the chord $W$ and becomes:

$$
F_{n}\left[\mathbf{M}_{L}(y), t\right]=W(y) F_{n}\left[\mathbf{M}_{o}, \mathbf{M}_{L}(y), t\right]
$$

By combining Eqs. (B.3), (B.5) and (20), steps 1 and 2 can be simplified to the computation of the following $1 \mathrm{D}$ integral:

$$
v_{n}\left(\mathbf{M}_{2}, t\right)=C^{\prime \prime \prime} v_{n}\left(\mathbf{M}_{0}\right) \frac{\mathrm{d}^{3}}{\mathrm{~d} t^{3}} \int_{L} W(y) g_{\alpha_{s}}\left(\mathbf{M}_{L}, \mathbf{M}_{2}, \alpha_{s}, t-t_{0}\right) \mathrm{d} L(y)
$$

Finally, the simplified model consists in:

(1) Computing step 1, i.e. with Eqs. (1-12) being omitted; the propagation from $S_{0}$ to $S_{1}$ is reduced to a simple time delay $t_{0}$.

(2) Assuming the inclination angle to be perfectly adjusted, according to Eq. (24).

(3) Replacing step 2 (i.e. Eq. (20)) by radiation from a linear source of length $L=D_{E}$ located at position $x_{L}$, centered at $y=0$ and weighted by the function $W(y)$ (shown in Fig. B.2(d)). 
The results presented in the core of this paper (Figs. 7 and 10) show that the simplified model indeed provides rather accurate results (with the exception of the waveform shape), and makes it possible to achieve substantially reduced computation times. This confirms that the hypothesis of nearly plane wave propagation between emitter and solid, expressed by Eq. (B.2) (and also commented in Section 5), is fully justified here.

It is straightforward to show that if the inclination angle does not fulfill the relationship given by Eq. (24), (i.e. $\beta_{e}<\sin ^{-1}\left(c_{f} / c_{R}\right)$ or $\beta_{e}>\sin ^{-1}\left(c_{f} / c_{R}\right)$ ), the travel time along the path $\overline{\mathbf{M}_{0} \mathbf{M}_{1} \mathbf{M}_{L}}$ will not be same for each point $\mathbf{M}_{o}$. The contributions $h_{\Psi}\left(\mathbf{M}_{o}, \mathbf{M}_{L}, t\right)$ will thus arrive at different instants, such that they will not be perfectly superimposed. This effect reduces the amplitude of the re-radiated SW and also increases the effective surface $S_{1}$ contributing to the creation of the SW (i.e. $S_{1}$ can no longer be replaced by line $\boldsymbol{L}$ ). This is the reason for which the maximum SW signal level (Fig. 15(c)) is obtained when $\beta_{e}=\beta_{o}$, and for which $\beta_{e}$ has such a significant influence on the waveform and spectrum of the field characteristics of the radiated SW field (Fig. 15(a)).

An example of the usefulness of the simplified model is shown in Fig. B3, where the axial field (equivalent to that studied in Fig. 7(b)) is computed for two receiver sizes and for different attenuations in the solid. Fig. B.3(a) clearly shows that the finite size of the receiver is associated with spatial averaging, which masks certain details of the axial distribution (the results for a point-like receiver are close to those reported in [92]). Fig. B.3(b) illustrates the influence of attenuation in the solid, and confirms that the results shown in Fig. 7(b) are dominated essentially by attenuation in the Plexiglas. This explains why typical near-field behavior (maximum close to $x / N=1$ ) is not observed in core of this paper. 


\section{Bibliography}

1. D.E. Chimenti, Review of air-coupled ultrasonic materials characterization. Ultrasonics, 2014. 54(7): p. 1804-1816.

2. M.C. Remillieux, B.E. Anderson, T.J. Ulrich, et al., Review of air-coupled transduction for nondestructive testing and evaluation. Acoustics Today, 2014. 10(3): p. 36-45.

3. M. Luukkala, P. Heikkila, and J. Surakka, Plate wave resonance - a contactless method. Ultrasonics, 1971. 9(4): p. 201-208.

4. D.W. Schindel and D.A. Hutchins, Applications of Micromachined Capacitance Transducers in Air-coupled Ultrasonics and Nondestructive Evaluation. IEEE Transactions on Ultrasonics, Ferroelectrics, and Frequency Control, 1995. 42(1): p. 51-58.
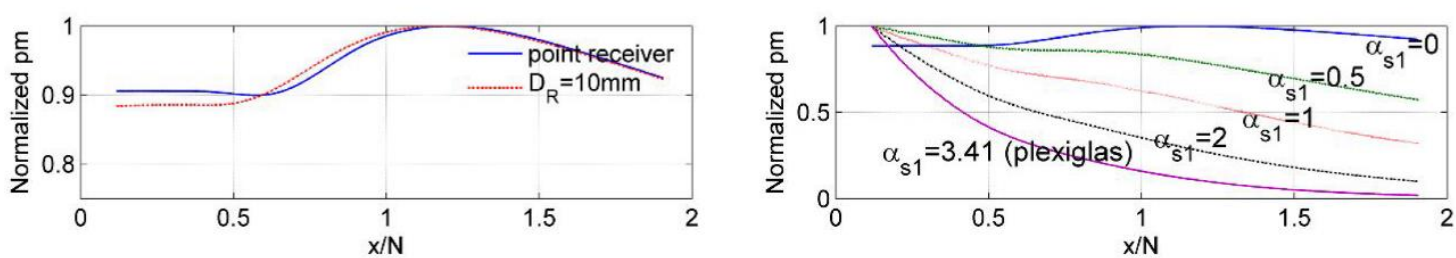

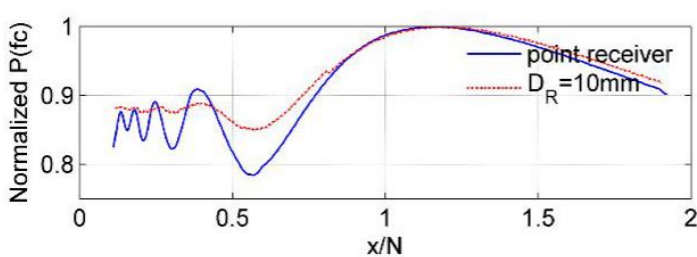

(a)

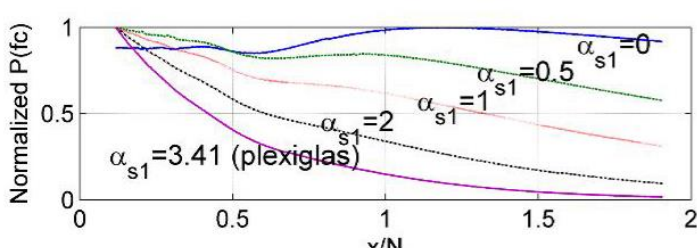

(b)

Fig. B.3. On-axis distribution computed using the simplified model for similar conditions as in Fig. 7(b). The upper plots show the results obtained from the time-domain signal $p_{m}(0.22<x / N<2, y=0)$, and the lower plots show the corresponding characteristics for a fixed-frequency $f_{o}=350 \mathrm{kHz}$. (a) Influence of the receiver dimension for a non-attenuating solid, for the point-like and finite-sized receivers considered in the core of this paper $\left(D_{R}=10 \mathrm{~mm}\right)$. (b) Influence of attenuation in the solid for a finite-sized receiver.

5. W.A. Grandia and C.M. Fortunko. NDE Applications of Air-coupled Ultrasonic Transducers. in IEEE Ultrasonics Symposium. 1995.

6. I. Solodov, K. Pfleiderer, H. Gerhard, et al., New opportunities for NDT with air-coupled ultrasound, in Emerging Technologies in NDT - Third International Conference. 2006. p. 176-183.

7. D.K. Hsu, D. Utrata, and M. Kuo. NDE of lumber and natural fiber based products with air coupled ultrasound. in Review of Progress in Quantitative Nondestructive Evaluation. 2010.

8. B. Piwakowski, M. Kaczmarek, and P. Safinowski. Evaluation of Concrete Cover by Surface Wave Technique: Identification Procedure. in AIP Conf. Proc. 2012. 
9. J. Li and B. Piwakowski, A time-domain model and experimental validation of the acoustic field radiated by air-coupled transducers. Ultrasonics, 2018. 82(1): p. 114-129.

10. H.-G. Li and Z.-G. Zhou, Application of P4 Polyphase codes pulse compression method to air-coupled ultrasonic testing systems. Ultrasonics, 2017. 78: p. 57-69.

11. Z.-G. Zhou, B.-Q. Ma, J.-T. Jiang, et al., Application of wavelet filtering and Barker-coded pulse compression hybrid method to air-coupled ultrasonic testing. Nondestructive Testing and Evaluation, 2014. 29(4): p. 297-314.

12. I.A. Victorov, Rayleigh and Lamb Waves: Physical Theory and Applications. 1967, New York: Plenum.

13. O. Abraham, B. Piwakowski, G. Villain, et al., Non-contact, automated surface wave measurements for the mechanical characterisation of concrete. Construction and Building Materials, 2012. 34: p. 904-915.

14. T.E. Michaels, J.E. Michaels, and M. Ruzzene, Frequency-wavenumber domain analysis of guided wavefields. Ultrasonics, 2011. 51: p. 452-466.

15. J.-Y. Zhu and J.S. Popovics, Non-contact NDT of Concrete Structures Using Air Coupled Sensors. 2008, Department of Civil and Environmental Engineering, University of Illinois at Urbana-Champaign.

16. H. Bjurstrom, N. Ryden, and B. Birgisson, Non-contact surface wave testing of pavements: comparing a rolling microphone array with accelerometer measurements. Smart Structures and Systems, 2016. 17(1): p. $1-15$.

17. H.M. Kim, I.K. Park, Y.K. Kim, et al. Identification of guided-wave mode in pipe by using air-coupled transducer. in Review of Quantitative Non-destructive Evaluation. 2005. AIP.

18. T.B. Hudson, T.-H. Hou, B.W. Grimsley, et al., Imaging of local porosity/voids using a fully non-contact air-coupled transducer and laser Doppler vibrometer system. Structural Health Monitoring, 2016: p. $1-10$.

19. M.S. Harb and F.-G. Yuan, Damage imaging using non-contact air-coupled transducer/laser Doppler vibrometer system. Structural Health Monitoring, 2016. 15(2): p. 193-203.

20. B. Piwakowski, A. Fnine, M. Goueygou, et al., Generation of Rayleigh waves into mortar and concrete samples. Ultrasonics, 2004. 42: p. 395-402.

21. R.J. Hallermeier and O.I. Diachok, Simple technique for exciting and probing elastic surface waves. Journal of Applied Physics, 1970. 41(12): p. 4763-4764.

22. R. Briers, O. Leroy, and G. Shkerdin, A liquid wedge as generating technique for Lamb and Rayleigh waves. Journal of Acoustic Society of America, 1997. 102(4): p. 2117-2124.

23. M. Castaings and P. Cawley, The generation, propagation, and detection of Lamb waves in plates using air-coupled ultrasonic transducers. Journal of Acoustic Society of America, 1996. 100(5): p. 3070-3077.

24. W. Ke, M. Castaings, and C. Bacon, 3D finite element simulations of an air-coupled ultrasonic NDT system. NDT\&E International, 2009. 42: p. 524-533.

25. Y.-K. An, B. Park, and H. Sohn, Complete noncontact laser ultrasonic imaging for automated crack visualization in a plate. Smart Materials and Structures, 2013. 22: p. 1-10.

26. S.K. Chakrapani, V. Dayal, and D. Barnard, Detection and characterization of waviness in unidirectional GFRP using Rayleigh wave air coupled ultrasonic testing. Research in Nondestructive Evaluation, 2013. 24: p. 191-201.

27. R. Raisutis, R. Kazys, E. Zukauskas, et al., Ultrasonic air-coupled testing of square-shape CFRP composite rods by means of guided waves. NDT \& E International, 2011. 44: p. 645-654.

28. B. Piwakowski and P. Safinowski, Non-destructive non-contact air-coupled concrete evaluation by an ultrasound automated device, in NDTCE' 09, Non-Destructive Testing in Civil Engineering. 2009: Nantes, France. 
29. J.-P. Balayssac, S. Laurens, G. Arliguie, et al., Description of the general outlines of the French project SENSO-Quality assessment and limits of different NDT methods. Construction and Building Materials, 2012. 35: p. 131-138.

30. V. Garnier, B. Piwakowski, and O. Abraham, Acoustic techniques for concrete evaluation: Improvements, comparisons and consistency. Construction and Building Materials, 2013. 43: p. 598-613.

31. V. Garnier, D. Martini, J. Salin, et al., Non-destructive testing of concrete: transfer from laboratory to on-site measurement, in 7th European Workshop on Structure Health Monitoring EWSHM 2014. 2014: Nantes, France.

32. V. Garnier, C. Payan, M. Lott, et al., Non Destructive Evaluation of containment walls in nuclear power plants, in 43rd Annual Review of Progress in Quantitative Nondestructive Evaluation. 2016. p. 080018-1-080018-9.

33. L. Ambrozinski, B. Piwakowski, T. Stepinski, et al., Application of air-coupled ultrasonic transducers for damage assessment of composite panels, in 6th European Workshop on Structural Health Monitoring. 2014. p. 1-8.

34. Q. Liu, B. Piwakowski, Z. Lafhaj, et al., Automated non-contact NDT by ultrasonic surface waves, in 2014 Far East Forum on Nondestructive Evaluation/Testing: New Technology and Application. 2014, IEEE: Chengdu, China.

35. M.S. Harb and F.G. Yuan, A rapid, fully non-contact, hybrid system for generating Lamb wave dispersion curves. Ultrasonics, 2015. 61: p. 62-70.

36. Z.-C. Fan, W.-T. Jiang, M.-L. Cai, et al., The effects of air gap reflections during air-coupled leaky Lamb wave inspection of thin plates. Ultrasonics, 2015.

37. S. Delrue, K.V.D. Abeele, E. Blomme, et al., Two-dimensional simulation of the single-sided air-coupled ultrasonic pitch-catch technique for non-destructive testing. Ultrasonics, 2010. 20: p. 188-196.

38. J.L. Prego-Borges, Lamb: a simulation tool for air-coupled Lamb wave based ultrasonic NDE systems. 2010, Polytechnic University of Catalonia UPC.

39. S. Banerjee and T. Kundu, Ultrasonic field modeling in plates immersed in fluid. Solids and Structures, 2007. 44: p. 6013-6029.

40. T. Kundu and D. Placko, Advanced Ultrasonic Methods for Material and Structure Inspection. 2007, United States: ISTE Ltd.

41. D. Placko and T. Kundu, DPSM for modeling Engineering Problems. 2007, New Jersey: John Wiley \& Sons, Inc.

42. S. Banerjee, T. Kundu, and N.A. Alnuaimi, DPSM technique for ultrasonic field modelling near fluid-solid interface. Ultrasonics, 2007. 46(3): p. 235-250.

43. T. Misaridis and J.A. Jensen, Use of modulated excitation signals in medical ultrasound. Part I: basic concepts and expected benefits. IEEE Transactions on Ultrasonics, Ferroelectrics, and Frequency Control, 2005. 52(2): p. 177-191.

44. H.E. Bass, L.C. Sutherland, and A.J. Zuckerwar, Atmospheric absorption of sound: Update. Journal of Acoustic Society of America, 1990. 88(4): p. 2019-2021.

45. L.B. Evans, H.E. Bass, and L.C. Sutherland, Atmospheric absorption of sound: theoretical predictions. Journal of Acoustic Society of America, 1971. 51(5): p. 1565-1575.

46. COMSOL, Acoustics Module User's Guide 5.0. 2014.

47. D. Guyomar and J. Powers, Boundary effects on trasient radiation fields from vibrating surfaces. Journal of Acoustic Society of America, 1984. 77(3): p. 907-915. 
48. T.E.G. Alvarez-Arenas, Acoustic impedance matching of piezoelectric transducers to the air. IEEE Transactions on Ultrasonics, Ferroelectrics, and Frequency Control, 2004. 51(5): p. 624-633.

49. M. Kaczmarek, B. Piwakowski, and R. Drelich, Non-Contact Ultrasonic Non-Destructive Techniques: State of the Art and Their Use in Civil Engineering. Journal of Infrastructure Systems, 2016: p. B4016003-1-11.

50. C.L. Pekeris, The seismic surface pulse. Geophysics, 1955. 41: p. 469-480.

51. S. Foti, Multistation methods for geotechnical characterization using surface waves. 2000, Politecnico di Torino.

52. F.R. Breckenridge and M. Greenspan, Surface-wave displacement: Absolute measurements using a capacitive transducer. Journal of Acoustic Society of America, 1981. 69(4): p. 1177-1185.

53. K. Aki and P.G. Richards, Quantitative Seismology, Theory and Methods. 1980, San Francisco: CA, Freeman.

54. J.H. F. Richart, R. Woods, Vibrations of soils and foundations. 1970, Englewood Cliffs, New Jersey: Prentice-Hall Inc.

55. A. Lhemery, A model for the trasient ultrasonic field radiated by an arbitrary loading in a solid. Journal of Acoustic Society of America, 1994. 96(6): p. 3776-3786.

56. P. Wilcox, R. Monkhouse, M. Lowe, et al., The use of Huygens' principle to model the acoustic field from interdigital Lamb wave transducers. Review of Progress in Quantitative Nondestructive Evaluation, 1998. 17: p. 915-922.

57. L.W. Schmerr-Jr. and A. Sedov, Ultrasonic beam models for the generation of surface waves and plate waves with angle beam transducers. Review of Progress in Quantitative Nondestructive Evaluation, 2011. 30: p. 771-778.

58. S.Z. Zhang, X.B. Li, H. Jeong, et al., Modeling linear Rayleigh wave sound fields generated by angle beam wedge transducers. AIP Advances, 2017. 7(1): p. 015005-1-13.

59. X.-Y. Zhao, L.W.S. Jr, A. Sedov, et al., Ultrasonic Beam Models for Angle Beam Surface Wave Transducers. Research in Nondestructive Evaluation, 2016. 27(3): p. 175-191.

60. P. He, Determination of ultrasonic parameters based on attenuation and dispersion measurements. Ultrasonic Imaging, 1998. 20: p. 275-287.

61. K.R. Waters, M.S. Hughes, J. Mobley, et al., On the applicability of Kramers-Kronig relations for ultrasonic attenuation obeying a frequency power law. Journal of Acoustic Society of America, 2000. 108(2): p. 556-563.

62. K.R. Waters, J. Mobley, and J.G. Miller, Causality-Imposed (Kramers-Kronig) Relatiohsips Between Attenuation and Dispersion. IEEE Transactions on Ultrasonics, Ferroelectrics, and Frequency Control, 2005. 52(5): p. 822-833.

63. K.R. Waters, M.S. Hughes, J. Mobley, et al., Differential forms of the Kramers-Kronig dispersion relations. IEEE Transactions on Ultrasonics, Ferroelectrics, and Frequency Control, 2003. 50(1): p. 68-76.

64. J.F. Kelly and R.J. McGough, Analytical time-domain Green's functions for power-law media. Journal of Acoustic Society of America, 2008. 124(5): p. 2861-2872.

65. J.F. Kelly and R.J. McGough, Causal impulse response for circular sources in viscous media. Journal of Acoustic Society of America, 2008. 123(4): p. 2107-2116.

66. C. Mattei and L. Adler, Leaky wave detection at air-solid interfaces by laser interferometry. Ultrasonics, 2000. 38: p. 570-574. 
67. H.L. Bertoni and T. Tamir, Unified theory of Rayleigh-angle phenomena for acoustic beams at liquid-solid interface. Applied Physics, 1973. 2: p. 157-172.

68. Ultragroup. Available from: www.ultrangroup.com.

69. Microacoustic. Available from: http://microacoustic.com/index.htm.

70. J. Krautkramer and H. Krautkramer, Ultrasonic Testing of Materials. 1990, New York: Springer-Verlag Berlin Heidelberg.

71. H. Ryan, Ricker, Ormsby, Klauder, Butterworth- A Choice of Wavelets. CSEG Recorder, 1994: p. 8-9.

72. P.D. Wilcox, A rapid signal processing technique to remove the effect of dispersion from guided wave signals. IEEE Transactions on Ultrasonics, Ferroelectrics, and Frequency Control, 2003. 50(4): p. 419-427.

73. J.E. Michaels, S.J. Lee, A.J. Croxford, et al., Chirp excitation of ultrasonic guided waves. Ultrasonics, 2013. 53: p. 265-270.

74. B. Piwakowski and K. Sbai, A New Approach to Calculate the Field Radiated from Arbitrarily Structured Transducer Arrays. IEEE Transactions on Ultrasonics, Ferroelectrics, and Frequency Control, 1999. 46(2): p. 422-440.

75. F. Lingvall, User Manual for the DREAM Toolbox Version 2.1.3. 2009.

76. L. Cartz, Nondestructive Teating-Radiography, Ultrasonics, Liquid Penetrant, Magentic Particle, Eddy Current. 1995, United States of America: ASM International.

77. R. Nave. Speed of Sound in Air. 2001; Available from: http://230nsc1.phy-astr.gsu.edu/hbase/Sound/souspe.html.

78. L.J. Bond, C.-H. Chiang, and C.M. Fortunko, Absorption of ultrasonic waves in air at high frequencies (10-20 MHz). Journal of Acoustic Society of America, 1992. 92(4): p. 2006-2015.

79. R. J. Hyndman and A.B. Koehler, Another look at measures of forecast accuracy. International Journal of Forecasting, 2006. 22(4): p. 679-688.

80. J. Zemanek, Beam Behavior within the Nearfield of a Vibrating Piston. Journal of Acoustic Society of America, 1970. 49: p. 181-191.

81. G. Taguchi, S. Chowdhury, and Y. Wu, Taguchi's Quality Engineering Handbook. 2007, Hoboken, New Jersey: John Wiley \& Sons, Inc.

82. M. Castaings, P. Cawley, R. Farlow, et al., Single Sided Inspection of Composite Materials Using Air Coupled Ultrasound. Journal of Nondestructive Evaluation, 1997. 17(1): p. 37-45.

83. S.J. Sanabria, T. Marhenke, R. Furrer, et al., Calculation of volumertric sound field of pulsed air-coupled ultrasound transducers based on single-plane measurements. IEEE Transactions on Ultrasonics, Ferroelectrics, and Frequency Control, 2018. 65(1): p. 72-84.

84. J.N. Tjotta and S. Tjotta, An analytical model for the nearfield of a baffled piston transducer. Journal of Acoustic Society of America, 1980. 68(1): p. 334-339.

85. M.A. Breazeale, L. Adler, and G.W. Scott, Interaction of ultrasonic waves incident at the Rayleigh angle onto a liquid-solid interface. Journal of Applied Physics, 1976. 48(2): p. 530-537.

86. M.A. Ruiz and P.B. Nagy, Diffraction correction for precision surface acoustic wave velocity measurements. Journal of Acoustic Society of America, 2002. 112(3): p. 835-842.

87. E.P. Papadakis, The Measurement of Ultrasonic Attenuation, in Physical Acoustics. 1990, ACADEMIC PRESS, INC.: Boston. p. 107-155.

88. M. Goueygou, F. Soltani, Z. Lafhaj, et al., Effect of geometrical diffraction on non-contact measurements of ultrasonic attenuation in cement-based materials, in Non-destructive Testing in Civil Engineering, NDTCE'09. 2009: Nantes, France. 
89. F. Press and J. Healy, Absorption of Rayleigh waves in low-loss medis. Journal of Applied Physics, 1957. 28(11): p. 1323-1325.

90. G.A. McMechan and M.J. Yedlin, Analysis of dispersive by wave field transformation. Geophysics, 1981. 46: p. 869-874.

91. L. Ambrozinski, B. Piwakowski, T. Stepinski, et al., Evaluation of dispersion characteristics of multimodal guided waves using slant stack transform. NDT \& E International, 2014. 68: p. 88-97.

92. J.L.S. Emeterio and L.G. Ullate, Diffraction impulse response of rectangular transducers. Journal of Acoustic Society of America, 1992. 92(2): p. 651-662. 